\title{
Probing the scattering of equivalent electroweak bosons
}

\section{Journal Article}

\section{Author(s):}

Borel, Pascal; Franceschini, Roberto; Rattazzi, Riccardo; Wulzer, Andrea

Publication date:

2012-06

\section{Permanent link:}

https://doi.org/10.3929/ethz-b-000056274

\section{Rights / license:}

Creative Commons Attribution 4.0 International

\section{Originally published in:}

Journal of High Energy Physics 2012(6), https://doi.org/10.1007/JHEP06(2012)122 


\title{
Probing the scattering of equivalent electroweak bosons
}

\author{
Pascal Borel, ${ }^{a}$ Roberto Franceschini, ${ }^{a, b}$ Riccardo Rattazzi ${ }^{a}$ and Andrea Wulzer ${ }^{c, d}$ \\ ${ }^{a}$ Institut de Théorie des Phénomènes Physiques, EPFL, \\ CH-1015 Lausanne, Switzerland \\ ${ }^{b}$ Department of Physics, University of Maryland, \\ College Park, MD 20742, U.S.A. \\ ${ }^{c}$ Dipartimento di Fisica e Astronomia and INFN — Sezione di Padova, \\ Via Marzolo 8, I-35131 Padova, Italy \\ ${ }^{d}$ Institute for Theoretical Physics, ETH Zurich, \\ 8093 Zurich, Switzerland \\ E-mail: pasc.borel@gmail.com, rfrances@umd.edu, \\ riccardo.rattazzi@epfl.ch, wulzer@itp.phys.ethz.ch
}

ABSTRACT: We analyze the kinematic conditions under which the scattering of equivalent massive spin-1 vector bosons factorizes out of the complete process. In practice, we derive the conditions for the validity of the effective $W$ approximation, proposed long ago but never established on a firm basis. We also present a parametric estimate of the corrections to the approximation and explicitly check its validity in two examples.

KEywords: Beyond Standard Model, Standard Model

ARXIV EPRINT: 1202.1904 


\section{Contents}

1 Introduction 1

2 Basic picture and explicit checks 3

2.1 Basic picture 3

2.2 Numerical comparison $\quad 7$

2.2.1 Corrections as a function of the hardness of the final state $W \quad 9$

2.2.2 Corrections as a function of the hardness of the jets 11

3 The equivalent $W$ boson $\quad 12$

$\begin{array}{ll}3.1 & \text { Axial gauge and Feynman diagrams } \\ \end{array}$

$\begin{array}{lll}3.2 & \text { Splitting amplitudes } & 15\end{array}$

\begin{tabular}{ll}
3.3 & Derivation of EWA \\
\hline
\end{tabular}

3.4 Corrections to EWA 22

$\begin{array}{ll}3.5 & \text { The example of } W W \text { scattering }\end{array}$

4 Conclusions and outlook $\quad 29$

\section{Introduction}

High-energy scattering among longitudinally polarized electroweak vector bosons $\left(W_{L}\right)$ is obviously the most direct probe of the dynamics of electroweak symmetry breaking (EWSB) ([1-10]; "Vector boson scattering at high mass" in [11]). If a light Higgs boson $(H)$ exists, then processes involving $H$, along with $W_{L}$, are similarly important [12]. Unfortunately $W$ beams are not available, and so we cannot, strictly speaking, directly study their collisions. However that should not be a problem of principle, as we know very well that, because of initial state radiation, the quanta that probe short distance physics in high energy collisions are all eminently virtual. So, for instance, even though quarks and gluons do not even exist as asymptotic states, we can still study their short distance interactions via the parton model, or, more formally, thanks to factorization theorems. With the intuition offered by the parton model, it is then natural to expect that at sufficiently high energy, much above the $W$ mass $m$, and with a proper selection of the kinematics of the final states, one should also be able to effectively test the collisions of $W$ bosons. Considering the prototypical reaction $q \bar{q} \rightarrow q^{\prime} \bar{q}^{\prime} W W$ one would expect that to be the case in the regime where the transverse momentum $P_{\perp}^{W}$ of the $W$ 's is much larger than that of the final state fermions $p_{\perp}$, that is where the latter are forwardly emitted. In that regime it seems intuitively obvious that the class of diagrams shown in figure 1a will dominate over all others, like for instance figure $1 \mathrm{~b}$, because the low virtuality $V_{1}$ and $V_{2}$ of the intermediate $W$, which characterizes the forward emission, parametrically enhances their 

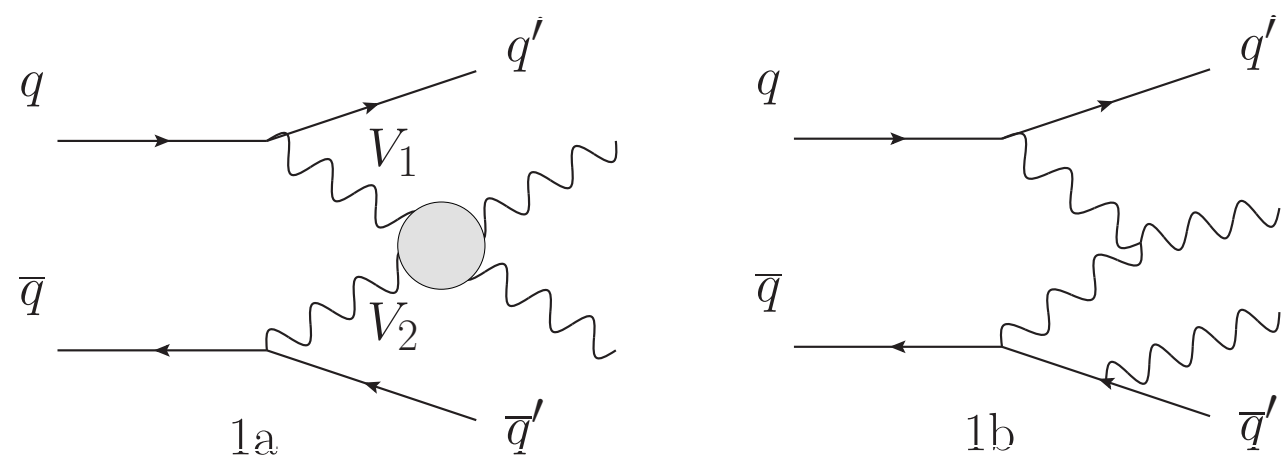

Figure 1. Diagrammatic contributions to the $q \bar{q} \rightarrow q^{\prime} \bar{q}^{\prime} W W$ process. On the left, the scattering topology. On the right, one representative "radiation" diagram.

propagators. Furthermore, in first approximation one expects to be able to neglect $V_{1,2}$ in the hard $W W \rightarrow W W$ subamplitude and to replace it with that for equivalent on-shell quanta. The differential cross section would then nicely factorize into a term describing $q \rightarrow q^{\prime} W$ splitting, which is controlled by known interactions, times the hard $W W \rightarrow W W$ scattering which is where new phenomena may well appear. This idea, inspired by the equivalent photon method [13-15], is known as the effective W approximation (EWA) [1624] and was largely employed in the 80's to simplify and speed up the computations of processes involving $W W \rightarrow X$. However, the conceptual validity of EWA was questioned by various authors, both in the early days and also more recently [25-27]. It is only in the case of a heavy Higgs, with strongly-coupled EWSB, that the issue has been set firmly, in favor of the validity of EWA (and factorization), in a very nice paper by Kunszt and Soper (KS) [28] in the late '80's. Later, the advent of powerful packages that allow to reliably and easily compute the exact cross section [29-35], including also radiative corrections [36, 37], has seemingly made EWA obsolete. Indeed, one often heard remark is that there is no way to single out diagrams with the scattering topology of figure 1a, and that all diagrams are equally important. We find that viewpoint disturbing for at least two reasons. On one side because it seems to entail that factorization fails for massive vector particles. On the other, because it suggests that it simply does not make sense, even in an ideal experimental situation, to extract in a model independent way the on-shell $\langle W W W W\rangle$ correlator from experimental data: the interesting physics of $W W$ scattering would always be mixed up in an intricate way with SM effects. We thus believe that studying the conditions for the applicability of EWA is important, and timely as well. Obviously the goal is not to find a fast and clever way to do computations. One should view EWA as a selection tool that allows to identify the relevant kinematic region of the complete process, the one which is more sensitive to the EWSB dynamics. One would want to focus on the kinematics where EWA applies not to speed up the computations, but to gain sensitivity to the relevant physics.

In this paper we shall analyze in detail the applicability of EWA. We will find, not surprisingly, that, in the proper kinematic regime, factorization is valid and EWA works 
egregiously. In order to prove that, we shall not need to focus, as KS did, on the case of a heavy Higgs or a strongly interacting EWSB sector, actually we shall not even need to restrict on the specific sub-process $W W \rightarrow W W$. Factorization indeed does not rely in any way on the detailed nature of the hard sub-process. It relies instead on the existence of a large separation of virtuality scales between the sub-process and the collinear $W$ emission. That only depends on kinematics and corresponds to requiring forward energetic jets and hard high $P_{\perp}$ outgoing $W$ 's. When those conditions are imposed EWA works well, for both longitudinally and transversely polarized $W$ 's, also including the case of weakly-coupled EWSB (light and elementary Higgs) where all helicities interact with the same strength $\sim g_{W}$ at all energies.

One serious issue in the applicability of EWA is the size of the subleading corrections. In practice one would like to know how well it can be applied at the LHC with $14 \mathrm{TeV}$ in the center of mass. A detailed quantitative answer to this question is beyond the scope of the present paper, we shall content ourselves with deducing a parametric estimate of the corrections. Our result is that typically the corrections scale quadratically with the inverse of the hardness $H$ of the $W W$ collision, and can be estimated as $\sim m^{2} / H^{2}$ and $p_{\perp}^{2} / H^{2}{ }^{1}$ We will give a numerical confirmation of this scaling, finding however a typically large numerical prefactor that enhances the corrections.

The paper is organized as follows. In section 2.1 we will introduce the EWA formula, discuss the basic physical reasons for its validity and state our results concerning the scaling of the corrections. In section 2.2 we will confirm these results in two examples, the processes $q W^{-} \rightarrow q^{\prime} W^{+} W^{-}$and $q \bar{q} \rightarrow q^{\prime} \bar{q}^{\prime} W^{+} W^{-}$, by comparing explicitly the exact cross section with its EWA approximation. Section 3 contains our analytical derivations, and constitutes the main part of the paper. In section 3.1 we introduce the technical tools that are needed in our derivation; in section 3.2 we compute the amplitude for the "soft" $q \rightarrow q^{\prime} W$ splitting and, finally, in section 3.3 we derive the EWA formula. We discuss the parametric estimate of the corrections in section 3.4, while section 3.5 illustrates some aspects of our derivation in the case of the $W W \rightarrow W W$ sub-process. Finally, we report our conclusions in section 4 .

\section{Basic picture and explicit checks}

While the general derivation of the EWA, which we will present in section 3 , is rather technical, the basic picture that underlies its validity is instead very simple and intuitive. In the present section we will first of all illustrate this picture and afterwards we will check the approximation explicitly in the relevant example of $W W \rightarrow W W$ scattering in the SM with a light Higgs. By the numerical comparison among the exact and the EWA cross sections we will also establish the scaling of the corrections to the approximation, a scaling which we will deduce analytically and cross-check in section 3 .

\subsection{Basic picture}

Let us consider a generic scattering process, of the type $q X \rightarrow q^{\prime} Y$, in which a massless quark (or a lepton) $q$ scatters on an unspecified initial particle $X$, gets converted into a

\footnotetext{
${ }^{1}$ Parametric enhancements are possible but only in very peculiar situations, as we shall discuss.
} 


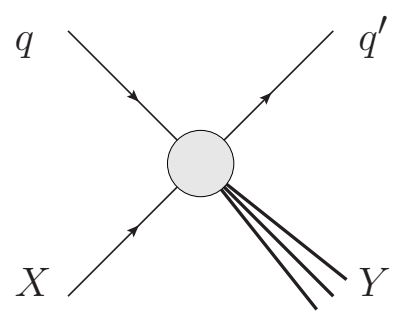

$$
\begin{gathered}
P_{q}=(E, \overrightarrow{0}, E) \quad P_{X}=\left(E_{X}, \overrightarrow{0},-E\right) \\
P_{q^{\prime}}=\left(\sqrt{(1-x)^{2} E^{2}+p_{\perp}^{2}}, \overrightarrow{p_{\perp}}, E(1-x)\right) \\
\vec{p}_{\perp}=\left\{p_{\perp} \cos \phi, p_{\perp} \sin \phi\right\}
\end{gathered}
$$

Figure 2. Pictorial representation of the $q X \rightarrow q^{\prime} Y$ process, which defines our parametrization of the quark momenta $P_{q}$ and $P_{q^{\prime}}$ in the center of mass frame. The momentum fraction $x$ is taken to be in the $(0,1)$ interval and far enough from the extremes in order for the first condition in eq. (2.2) to be satisfied.

second quark $q^{\prime}$ possibly of different charge and produces an unspecified final state $Y$. The processes we are interested in are the "weak" ones, in which the only relevant interaction of the quarks is provided by the standard minimal weak coupling with the $W$ bosons. To simplify the discussion we ignore the QCD interactions and set to zero the hypercharge coupling $g^{\prime}$. In practice, we assume that the only relevant quark vertex is given by

$$
\mathcal{L}_{q}=\frac{g}{2} \bar{q}_{L} \sigma^{a} \gamma^{\mu} q_{L} W_{\mu}^{a}
$$

We see no obstruction to include hypercharge vertices and we do not expect any qualitative change in the conclusions.

Apart from the minimality of the quark's interaction in eq. (2.1), which however is very well motivated experimentally and therefore does not constitute a limitation, ${ }^{2}$ we will not need any other assumption on the theory that governs the dynamics of the weak bosons. Given that we aim, as explained in the Introduction, to use EWA as a tool for probing the presently unknown EWSB sector, it is important to stress (and to prove, as we will do in section 3) that the validity of EWA does not rely on any assumption on EWSB. The sector responsible for the $W$ interactions is generic and, given the typical energy $E$ of the scattering process, can be depicted as a set of particles with mass below or around $E$ that interact through a set of couplings $c_{i}$, possibly originating by integrating out states that are much heavier than $E$. For example, suppose that the EWSB sector just consists of the standard Higgs model. If the Higgs is light $\left(m_{H} \lesssim E\right)$, the appropriate description of the $W \mathrm{~s}$ and Higgs interactions is provided by the linear Higgs Lagrangian, and only contains renormalizable couplings. If on the contrary the Higgs is heavy $\left(m_{H} \gg E\right)$, the $W$ interactions are dictated by the non-renormalizable $\sigma$-model obtained by integrating it out; our general analysis will encompass both possibilities.

The essence of EWA is that the complete $q X \rightarrow q^{\prime} Y$ process is equivalent, at high enough energies and under particular kinematic conditions, to the $W X \rightarrow Y$ hard subprocess initiated by an on-shell $W$ boson, convoluted with the splitting function that describes the collinear emission $q \rightarrow q^{\prime} W$. EWA is a particular example of the general

\footnotetext{
${ }^{2}$ In the kinematic regime where EWA applies, the $W q \bar{q}$ interaction is probed at a low virtuality where the possible effect of new physics, parametrized by higher dimension operators, is negligible.
} 


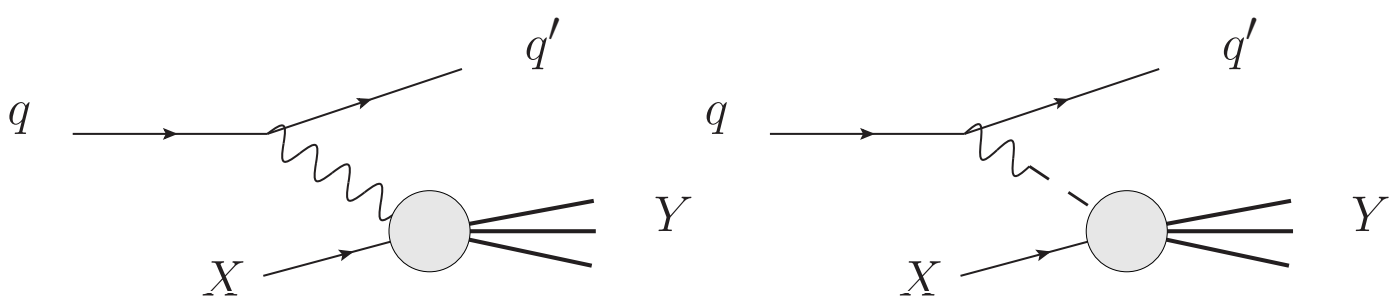

Figure 3. The topology of the scattering contributions to the $q X \rightarrow q^{\prime} Y$ process, the second diagram contains a mixed gauge-goldstone propagator which arises because we work in the axial gauge.

phenomenon of factorization which typically takes place in the limit of highly energetic and forward splitting. We therefore expect EWA to hold when the energy $E$ of the process is much larger than the mass $m$ of the $W$ and in the special kinematic regime in which the quark undergoes, in the center of mass of the collision, a small angular deflection while losing a sizable fraction of its initial energy. More precisely, in the parametrization of the momenta defined by figure 2, the regime relevant for EWA is

$$
E \sim x E \sim(1-x) E, \quad \delta_{m} \equiv \frac{m}{E} \ll 1, \quad \delta_{\perp} \equiv \frac{p_{\perp}}{E} \ll 1,
$$

where no assumption is made, a priori, on the relative size of $p_{\perp}$ and $m$. In that kinematic regime, let us consider, among the different diagrams contributing to $q X \rightarrow q^{\prime} Y$, the "scattering" diagrams depicted in figure 3. At the $W \bar{q}^{\prime} q$ vertex, on-shell $q$ and $q^{\prime}$ are respectively annihilated and created, together with the creation of an off-shell virtual $W$ of momentum

$$
K \equiv P_{q}-P_{q^{\prime}}=\left(\sqrt{x^{2} E^{2}+p_{\perp}^{2}+m^{2}-V^{2}},-\overrightarrow{p_{\perp}}, x E\right)
$$

where the virtuality (off-shelness) $V$ is given by

$$
V^{2}=m^{2}-K^{2} \simeq m^{2}+\frac{p_{\perp}^{2}}{1-x}\left[1+\mathcal{O}\left(\frac{p_{\perp}}{E}\right)^{2}\right]
$$

Once emitted, the virtual $W$ enters the $W X \rightarrow Y$ sub-process, which we assume to be characterized by a hardness $H$ of order $E$. The hardness corresponds to the time scale of the $W X \rightarrow Y$ sub-process. The virtuality $V$, instead, sets the time interval $\Delta T$ during which the virtual $W$ is indistinguishable, due the Uncertainty Principle, from an on-shell physical $W$. The correction to the $W$ energy due to off-shellness is indeed $\Delta E \sim V^{2} / E$, from which $\Delta T \sim E / V^{2}$. Given that $V$ is much smaller than $E\left(V^{2} / E^{2}=\mathcal{O}\left(\delta_{m}^{2}+\delta_{\perp}^{2}\right)\right)$ because of eq. (2.2), the time scale $1 / E$ of the $W X \rightarrow Y$ sub-process is much shorter than $\Delta T$ meaning that it must be possible to regard the $W$ as an on-shell particle during the hard scattering. The full process must factorize in terms of the on-shell $W X \rightarrow Y$ cross section.

Technically, EWA can be stated as follows $[16,17]$. Consider the exact cross-section $d \sigma\left(q X \rightarrow q^{\prime} Y\right)$, integrated over the azimuthal angle $\phi$ (see figure 2) of the final quark but 
completely differential in all the other kinematic variables. In the $\delta_{m, \perp} \rightarrow 0$ limit, according to EWA the unpolarized cross-section $d \sigma\left(q X \rightarrow q^{\prime} Y\right)$ reduces to

$$
\begin{aligned}
\frac{d \sigma_{\mathrm{EWA}}\left(q X \rightarrow q^{\prime} Y\right)}{d x d p_{\perp}}=\frac{C^{2}}{2 \pi^{2}}\left\{f_{+}\right. & \left.x, p_{\perp}\right) \times d \sigma\left(W_{+}^{Q} X \rightarrow Y\right) \\
+ & f_{-}\left(x, p_{\perp}\right) \times d \sigma\left(W_{-}^{Q} X \rightarrow Y\right) \\
+ & \left.f_{0}\left(x, p_{\perp}\right) \times d \sigma\left(W_{0}^{Q} X \rightarrow Y\right)\right\},
\end{aligned}
$$

where each line is associated with the exchange of an equivalent $W$ boson of a different polarization $r= \pm, 0$. The three splitting functions $f_{ \pm}$and $f_{0}$ describe the collinear emission of the polarized equivalent $W$ and $d \sigma\left(W_{r}^{Q} X \rightarrow Y\right)$ denotes the totally differential polarized cross-section of the $W_{r}^{Q} X \rightarrow Y$ hard sub-process. In the sub-process the equivalent $W_{r}^{Q}$ is treated as a perfectly physical on-shell particle of four-momentum

$$
K_{W}=\left(\sqrt{x^{2} E^{2}+m^{2}}, \overrightarrow{0}, x E\right) .
$$

The variable $x$ thus corresponds to the fraction of longitudinal momentum (which approximately coincides with the energy for $E \gg m$ ) carried away by the equivalent $W$ from the fermionic line. Explicitly, the splitting functions are given by

$$
\begin{aligned}
f_{+}\left(x, p_{\perp}\right) & =\frac{(1-x)^{2}}{x} \frac{p_{\perp}^{3}}{\left(m^{2}(1-x)+p_{\perp}^{2}\right)^{2}}, \\
f_{-}\left(x, p_{\perp}\right) & =\frac{1}{x} \frac{p_{\perp}^{3}}{\left(m^{2}(1-x)+p_{\perp}^{2}\right)^{2}}, \\
f_{0}\left(x, p_{\perp}\right) & =\frac{(1-x)^{2}}{x} \frac{2 m^{2} p_{\perp}}{\left(m^{2}(1-x)+p_{\perp}^{2}\right)^{2}} .
\end{aligned}
$$

The label $Q= \pm 1,0$ appearing in the previous equations denotes the electric charge difference between $q$ and $q^{\prime}$ and obviously corresponds to the charge of the equivalent $W$ emitted in the splitting. Depending on $Q$, the coefficient $C$ in eq. (2.5) reads

$$
\begin{array}{lll}
C=\frac{g}{4} & \text { for } & Q=0, \\
C=\frac{g}{2 \sqrt{2}} & \text { for } & Q= \pm 1 .
\end{array}
$$

For processes initiated by two quarks, or by a quark and an antiquark $q_{i} \bar{q}_{j} \rightarrow q_{i}^{\prime} \bar{q}_{j}^{\prime} X$ which both collinearly produce two equivalent $W$ 's, EWA straightforwardly generalizes to

$$
\frac{d \sigma_{\mathrm{EWA}}\left(q_{i} \bar{q}_{j} \rightarrow q_{i}^{\prime} \bar{q}_{j}^{\prime} X\right)}{d x_{i} d x_{j} d p_{\perp, i} d p_{\perp, j}}=\sum_{r, s} \frac{C_{i}^{2}}{2 \pi^{2}} \frac{C_{j}^{2}}{2 \pi^{2}} f_{r}\left(x_{i}, p_{\perp, i}\right) f_{-s}\left(x_{j}, p_{\perp, j}\right) \times d \sigma\left(W_{r}^{Q_{i}} W_{s}^{Q_{j}} \rightarrow X\right)
$$

where the inversion of the equivalent $W$ polarization in the anti-quark splitting function follows from $C P$ conjugation.

The derivation of eq. (2.5), which we will describe in section 3 , basically consists of a Laurent series expansion of the exact scattering amplitude in the parameters $\delta_{\perp}=p_{\perp} / E$ 
and $\delta_{m}=m / E$, a procedure that will also allow us to establish the scaling of the corrections to the approximation. We will find that the relative corrections are typically quadratic in $\delta_{m}$ and in $\delta_{\perp}$, i.e.

$$
\delta_{\mathrm{EWA}} \simeq \max \left[\delta_{m}^{2}, \delta_{\perp}^{2}\right],
$$

even though they can be enhanced in some particular condition as we will discuss in section 3.4. For instance, in the very low- $p_{\perp}$ region $p_{\perp} \ll m$ the error scales in some cases as $\delta_{\mathrm{EWA}} \simeq\left(m^{2} / p_{\perp} E\right)^{2}$ and in particular for $p_{\perp} \simeq m^{2} / E$ EWA fails even if $\delta_{m}$ and $\delta_{\perp}$ are extremely small. We will confirm the above result in the following section, by computing the corrections explicitly in the case of the $W W \rightarrow W W$ hard sub-process.

Before moving forward, two important comments are in order. The first is that for the intuitive derivation of the EWA previously discussed it has been crucial to assume that the $W X \rightarrow Y$ sub-process is genuinely hard, with a hardness $H \sim E$ and that it contains no other softer scales. This excludes, for instance, the case where the momenta of the final particles $Y$ become soft, or collinear among each other, with $P_{X}$ or with the virtual $W$ momentum $K$. In order to apply EWA all those kinematic configurations must be avoided by suitable cuts on the $Y$ momenta. In the processes considered in the following section, for instance, the region of forward $W W \rightarrow W W$ scattering is soft because it is characterized by a low value of the $t$ variable and will have to be excluded by a hard cut on the transverse momentum $P_{\perp}^{W}$ of the final $W$ 's. Moreover, given that factorization relies on the hierarchy among the virtuality of the soft splitting and the hardness $H$ of the sub-process, one might expect that a numerically more precise estimate of the corrections to the EWA could be obtained by comparing $p_{\perp}$ and $m$ with $H$ instead of $E$, i.e. by redefining $\delta_{m}=m / H$ and $\delta_{\perp}=p_{\perp} / H$. We will verify this expectation in the following section, where $H=P_{\perp}^{W}$.

The second comment is that the intuitive argument in favor of the EWA implicitly relied on a gauge choice because of its starting point, which consisted in interpreting the $W$ propagator as the exchange of off-shell but otherwise perfectly physical $W$ quanta. This interpretation is only valid in "physical" gauges while in a generic one, including for instance the covariant gauges, extra unphysical states propagate and the scattering diagrams are not endowed with the physically transparent interpretation outlined above. This suggests that the task of providing a technical proof of the EWA might be easier to accomplish if working in a physical gauge, and this is indeed what we will do in section 3, where we will choose the axial gauge.

\subsection{Numerical comparison}

In order to get a first confirmation of the validity of the EWA approximation in eqs. (2.5), (2.9), we will now perform a numerical comparison of $d \sigma_{\mathrm{EWA}}$ with the exact differential cross section $d \sigma_{E X A C T}$ in some explicit examples. We will quantify the accuracy of the approximation by computing

$$
\delta_{\mathrm{EWA}} \equiv 2 \frac{d \sigma_{E X A C T}-d \sigma_{\mathrm{EWA}}}{d \sigma_{E X A C T}+d \sigma_{\mathrm{EWA}}},
$$

at some reference points in phase space. We will consider two processes: the $2 \rightarrow 3$

$$
u W^{-} \rightarrow d W^{+} W^{-} .
$$


and the $2 \rightarrow 4$ quark/antiquark scattering

$$
u \bar{c} \rightarrow d \bar{s} W^{+} W^{-},
$$

where we have chosen initial quarks of different families (we work at vanishing Cabibbo angle) just for simplicity, in order to avoid a proliferation of diagrams with $q \bar{q}$ annihilation.

Let us describe the kinematics for the approximated and for the exact cross section. The hard $2 \rightarrow 2$ cross section which appears in EWA, $d \sigma\left(W_{r}^{+} W_{s}^{-} \rightarrow W^{+} W^{-}\right)$, is computed by taking as independent kinematic variables the polar angle $\theta$ in the center of mass (C.M.) of the collision and the azimuthal angle $\phi_{W}$ of the outgoing $W^{+}$. The C.M. energy of the $W^{+} W^{-}$system is instead fixed by the momenta of the incoming equivalent $W$ 's which, in analogy with eq. (2.6), read

$$
\begin{array}{lll}
\text { for the } 2 \rightarrow 3: & K_{+}=\left(\sqrt{m^{2}+E^{2} x^{2}}, 0,0, E x\right), \quad K_{-}=\left(\sqrt{m^{2}+E^{2}}, 0,0,-E\right), \\
\text { for the } 2 \rightarrow 4: & K_{+}=\left(\sqrt{m^{2}+E^{2} x^{2}}, 0,0, E x\right), \quad K_{-}=\left(\sqrt{m^{2}+E^{2} y^{2}}, 0,0,-E y\right),
\end{array}
$$

where $y$ is the energy fraction of the equivalent $W^{-}$coming from the anti-quark splitting. The remaining relevant kinematic variables characterizing the $2 \rightarrow 3$ and $2 \rightarrow 4$ processes (see eqs (2.5) and (2.9)) are just the absolute values of the outgoing quarks transverse momenta. These are the same as for the kinematics of the exact process that we describe below. In EWA one integrates on the quarks' azimuthal angles, and thus the result does not depend on $\phi_{W}$.

The kinematics of the exact $2 \rightarrow 4$ process is parametrized as follows. The incoming quark and anti-quark momenta are

$$
\begin{aligned}
P_{u} & =(E, 0,0, E), \\
P_{\bar{c}} & =(E, 0,0,-E),
\end{aligned}
$$

while for the outgoing ones we have, in analogy with figure 2,

$$
\begin{aligned}
& P_{d}=\left(\sqrt{E^{2}(1-x)^{2}+p_{\perp}^{2}}, p_{\perp} \sin (\phi), p_{\perp} \cos (\phi), E(1-x)\right), \\
& P_{\bar{s}}=\left(\sqrt{E^{2}(1-y)^{2}+q_{\perp}^{2}}, q_{\perp} \sin (\psi), q_{\perp} \cos (\psi),-E(1-y)\right) .
\end{aligned}
$$

In order to compare with EWA we must however integrate over the azimuthal angles of the outgoing quarks $\phi$ and $\psi$. As for the outgoing $W^{+} W^{-}$system, we describe it, as before, by the angles $\theta$ and $\phi_{W}$ of the $W^{+}$in the $W$-pair center of mass frame. Notice that now, unlike for the $2 \rightarrow 2$ EWA case, the boost of the $W^{+} W^{-}$system is not directed along the $z$-axis but has a transverse component recoiling against the quark transverse momenta $p_{\perp}$ and $q_{\perp}$. Nevertheless, by azimuthal symmetry, after integrating over $\phi$ and $\psi$, the remaining dependence on $\phi_{W}$ is trivial.

As for the exact $2 \rightarrow 3$ process, we describe the quark kinematics by the same $P_{u}$ and $P_{d}$ of eqs. (2.15), (2.17). In strict analogy we then define the $W W$ system variables 
and integrate over the $d$-quark azimuthal angle $\phi$. In the end, for the $2 \rightarrow 3$ process the independent kinematic variables we fix in order to compute $\delta_{\mathrm{EWA}}$ are $p_{\perp}, x$ and $\theta$. For the $2 \rightarrow 4$ process there are two additional variables $y, q_{\perp}$ associated with the $\bar{c} \rightarrow \bar{s}$ quark line. The dependence on $\phi_{W}$ is trivial. It should be noticed that the comparison between EWA and the exact cross section is not performed at exactly coincident kinematic points. This is because in the kinematics of EWA the conservation of both transverse momentum and energy is violated by the quark splitting into the equivalent vector bosons (eq. (2.14)). So at corresponding values of $\theta$ and $\phi_{W}$ the outgoing $W 4$-momenta differ in EWA and in the exact kinematics. The differences are however of order $p_{\perp}^{2} / E^{2}, m^{2} / E^{2}$. That is precisely the order at which we expect the approximation to hold.

Two final comments on the analysis that follows are in order. We shall also use a variable $k_{T}$, function of the independent kinematic variables, which corresponds to the transverse momentum of the outgoing $W^{+}$in EWA kinematics. By defing $s_{W W}=\left(K_{-}+\right.$ $\left.K_{+}\right)^{2}$ the CM square energy in the EWA kinematics we have

$$
k_{T}=\sin \theta \sqrt{\frac{s_{W W}-4 m^{2}}{4}} .
$$

Of course this quantity, as a function of $\theta, x, p_{\perp}\left(y, q_{\perp}\right)$ differs from the outgoing transverse momenta of either $W$ 's in the exact kinematics by an amount $O\left(\delta_{\perp}^{2}, \delta_{m}^{2}\right)$. We will call this variable the pseudo-transverse-momentum. In all the analysis we shall pick a reference point where the Higgs boson $H$ has a mass $m_{H}=2 m$ and consider vanishing hypercharge, compatibly with eq. (2.1). The scattering amplitudes are computed with the FeynArts/FormCalc system [38-40] and the numerical integration over the jets' azimuthal angle is performed using the CUBA library [41].

\subsubsection{Corrections as a function of the hardness of the final state $W$}

As explained in section 2.1, a necessary condition for factorization is a hierarchy between the virtuality (or the hardness) of the $q \rightarrow q^{\prime} W^{\prime}$ splitting and that of the $W W \rightarrow W W$ subprocess. As such we compute $\delta_{\mathrm{EWA}}$ as a function of the pseudo-tranverse-momentum $k_{T}$, by keeping the C.M. scattering angle $\theta$ and the outgoing jets transverse momenta fixed while increasing the center of mass energy. In particular for the process in eq. (2.12) we show our computation of $\delta_{\mathrm{EWA}}$ as a function of $k_{T} / m$ in figure 4 for a fixed kinematics given by

$$
p_{\perp}=45 \mathrm{GeV}, \quad x=0.65, \quad \sin \theta=0.95 .
$$

As we are interested to the general behavior of the accuracy of EWA rather than to what happens for each exclusive helicity channel the figure shows $\delta_{\mathrm{EWA}}$ for 3 out of the 27 possible helicity configurations of the external states of the process. The selected helicity configurations are the one with all longitudinal external states, which is interesting per se, and the two lines corresponding to the maximal and minimal $\delta_{\mathrm{EWA}}$ for the specified kinematics. The lines not shown do not add significant information for our analysis as they are very similar in shape to those in the figure. However it is important to stress that we see that for all helicity configurations there is convergence, which shows that EWA 


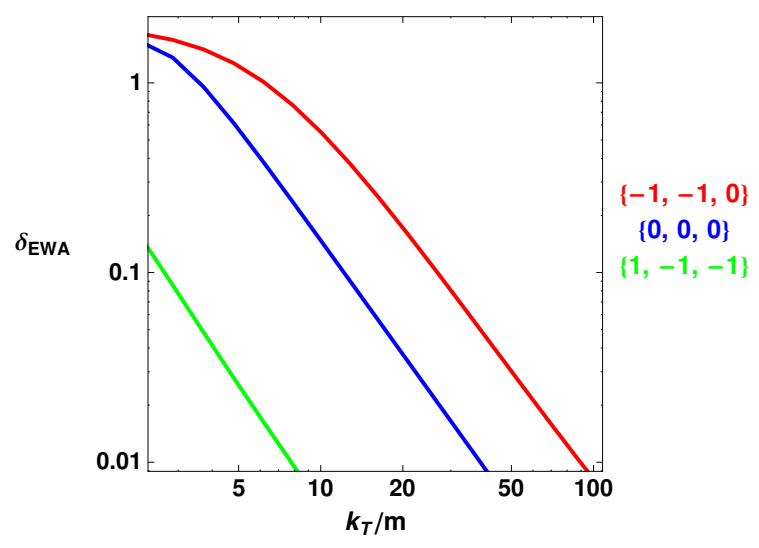

Figure 4. Accuracy of EWA for the $2 \rightarrow 3$ process in eq. (2.12) as a function of $k_{T} / m$ for fixed kinematics given by eq. (2.20). The helicities of the external $W$ bosons in each process are $\left\{\lambda\left(W_{\text {in }}^{-}\right), \lambda\left(W_{\text {out }}^{+}\right), \lambda\left(W_{\text {out }}^{-}\right)\right\}$as indicated by the colored labels at the right of the plot.

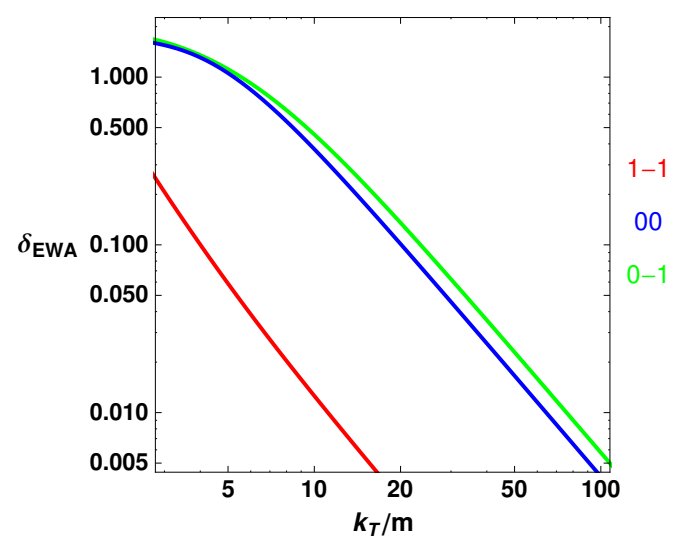

Figure 5. Accuracy of EWA for the $2 \rightarrow 4$ process eq. (2.13) as a function of $k_{T} / m$ for fixed kinematics given by eq. (2.21). The helicities of the external $W$ bosons in each process are $\left\{\lambda\left(W_{\text {out }}^{+}\right), \lambda\left(W_{\text {out }}^{-}\right)\right\}$as indicated by the colored labels at the right of the plot.

captures the leading behavior of the exact cross-section. Indeed from the figure one can read that $\delta_{\mathrm{EWA}}$ scales like $1 / k_{T}^{2}$, in agreement with the expectations. Notice however that the corrections are numerically larger than the estimate $\delta_{\mathrm{EWA}} \simeq m^{2} / k_{T}^{2}$ in eq. (2.10) (as $p_{\perp}<m$ in eq. (2.20) the corrections are dominated by $\left.m\right)$. This signals the presence of a large numerical coefficient, typically of the order of a few. Indeed for fixed $k_{T}$ there is an order of magnitude spread between the minimal and maximal $\delta_{\mathrm{EWA}}$ in figure 4 . It would be interesting to investigate the impact of this numerical enhancement in an experimentally realistic situation like the $\mathrm{LHC}$ at $14 \mathrm{TeV}$.

The analogous computation of $\delta_{\mathrm{EWA}}$ for the process in eq. (2.13) is shown in figure 5 for a fixed kinematics given by

$$
p_{\perp}=20 \mathrm{GeV}, q_{\perp}=10 \mathrm{GeV}, \quad x=0.4, \quad y=0.6, \quad \sin \theta=\frac{\sqrt{3}}{2} .
$$




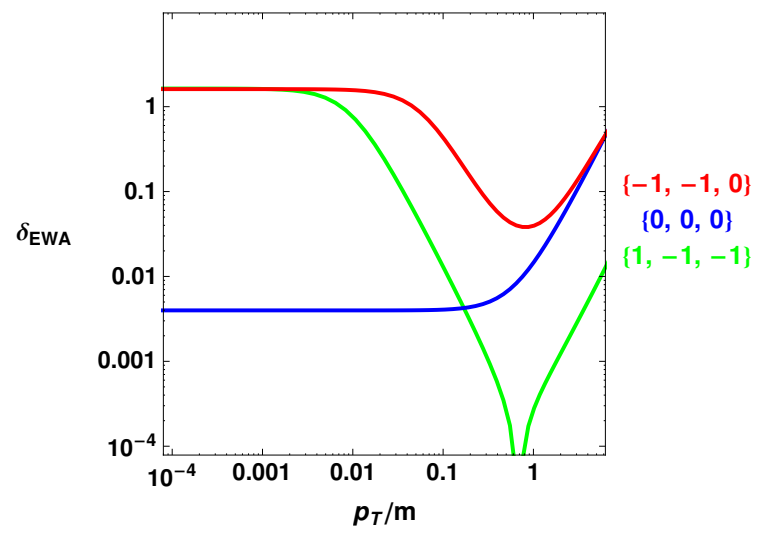

Figure 6. Accuracy of EWA as a function of $p_{T} / m$ for fixed kinematics as in eq. (2.22). The helicities of the external $W$ bosons in each process are $\left\{\lambda\left(W_{\text {in }}^{-}\right), \lambda\left(W_{\text {out }}^{+}\right), \lambda\left(W_{\text {out }}^{-}\right)\right\}$as indicated by the colored labels at the right of each plot.

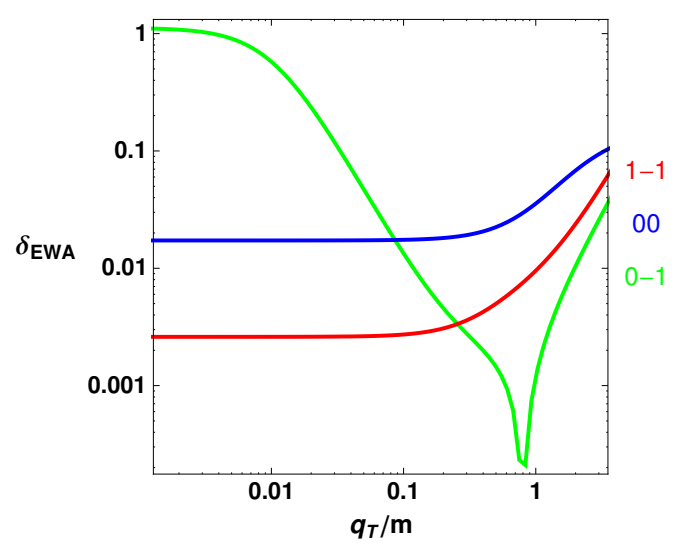

Figure 7. Accuracy of EWA as a function of $q_{\perp} / m$ for fixed kinematics given in eq. (2.23). The helicities of the external $W$ bosons in each process are $\left\{\lambda\left(W_{\text {out }}^{+}\right), \lambda\left(W_{\text {out }}^{-}\right)\right\}$as indicated by the colored labels at the right of the plot.

In the figure we show the line corresponding to longitudinal final state $W$ bosons and the two lines corresponding to maximal and minimal $\delta_{\mathrm{EWA}}$. We note that also for this process the scaling of the corrections to the EWA is such that $\delta_{\mathrm{EWA}} \sim k_{T}^{-2}$, in agreement with eq. (2.10).

\subsubsection{Corrections as a function of the hardness of the jets}

The results shown so far are relative to points of phase-space where, according to eq. (2.10), the corrections to EWA are dominated by $m$, as the chosen transverse momenta of the jets in eqs. (2.20) and (2.21) are relatively low. To fully check the structure of the corrections in eq. (2.10) we also have to study the dependence of $\delta_{\text {EWA }}$ on the quark transverse momenta. For the $2 \rightarrow 3$ process we perform this study by fixing

$$
E=10 \mathrm{TeV}, \quad x=0.65, \quad \sin \theta=0.86,
$$


and by computing $\delta_{\mathrm{EWA}}$ as a function of $p_{\perp} / m$. The results for the same helicity configurations of the previous section are reported in figure 6 . We notice, first of all, that $\delta_{\mathrm{EWA}}$ grows quadratically with $p_{\perp}$ in the region $p_{\perp}>m$. In that region, as predicted by eq. (2.10), the corrections must indeed go like $p_{\perp}^{2} / k_{T}^{2}$. At low $p_{\perp} \ll m$ different behaviors are observed for different helicities of the external $W$ bosons. For the process with three longitudinal $W$ bosons $\delta_{\text {EWA }}$ stabilizes to a constant value $\simeq m^{2} / k_{T}^{2}$. For the two other cases $\delta_{\text {EWA }}$ scales like $1 / p_{\perp}^{2}$ and for $p_{\perp} \simeq m^{2} / k_{T}$ EWA fails completely. The corrections can be estimated in this case as $\delta_{\mathrm{EWA}} \simeq\left(\mathrm{m}^{2} / p_{\perp} E\right)^{2}$ as anticipated in the discussion below eq. (2.10). We will explain this behavior in great detail in section 3.4 and 3.5. For the moment we anticipate that the only helicity channels for which $\delta_{\mathrm{EWA}}$ is enhanced are those dominated by the exchange of a transverse equivalent $W$. The enhancement of the relative corrections at low $p_{\perp}$ follows from the $p$-wave suppression of the leading order which affects the transverse splitting functions in eq. (2.7). For the $2 \rightarrow 4$ process we perform the same check by fixing

$$
E=10 \mathrm{TeV}, \quad p_{\perp}=20 \mathrm{GeV}, \quad x=0.4, \quad y=0.6, \quad \sin \theta=0.8,
$$

and compute $\delta_{\text {EWA }}$ as a function of $q_{\perp} / m$. The result is shown in figure 7 for the representative cases of the helicity configurations of the previous section. As well as in the case $2 \rightarrow 3$ we observe the presence of two behaviors at low $p_{\perp}$.

\section{The equivalent $\mathrm{W}$ boson}

The results of the previous section provide an extremely non-trivial confirmation of EWA, but of course their validity is limited to a specific process, $W W$ scattering, in a specific model of EWSB, the Higgs model. The aim of the present section is to overcome these limitations by deriving the EWA formula analytically and showing that its validity is neither restricted to a specific class of scattering processes nor to a specific model of EWSB.

Before proceeding it is worth recalling what is the main methodological obstacle in establishing EWA, the one that often causes confusion. When working with Feynman diagrams, the derivation of EWA, or of any factorization based on virtual quanta, amounts to trashing some diagrams and approximating others. However individual Feynman diagrams do not have absolute physical meaning, as their functional form and their numerical value depend on the chosen parametrization of the fields and, in particular, on the choice of gauge. In an arbitrary gauge, each trashed diagram, or the neglected off-shell piece in the diagrams of Figure 1a, can be arbitrarily sizeable, making the validity of the approximation not manifest at all. Of course the existence of a gauge where all the neglected terms are one by one numerically negligible would be sufficient to establish the approximation in all other gauges as well. To better appreciate how EWA stands as regards the above issue, it is sufficient to focus on the diagram in figure 1a. In an obvious notation it can be written as (see also the discussion in section 3.3)

$$
\left\{J_{\mu}^{1}\left(V_{1}^{2}\right) \frac{P^{\mu \nu}\left(\vec{p}_{1}, V_{1}^{2}\right)}{p_{1}^{2}-m^{2}}\right\}\left\{J_{\rho}^{2}\left(V_{2}^{2}\right) \frac{P^{\rho \sigma}\left(\vec{p}_{2}, V_{2}^{2}\right)}{p_{2}^{2}-m^{2}}\right\} \mathcal{A}_{\nu \sigma}\left(V_{1}^{2}, V_{2}^{2}\right)
$$


where we have highlighted the dependence of the amplitude for the $W W \rightarrow W W$ subprocess on the virtuality $V_{i}^{2} \equiv m^{2}-p_{i}^{2}$ of the intermediate $W$ 's. The above contribution to the total amplitude has a (pole) singularity at $V_{i}=0$. More importantly, it is the only contribution featuring such a singularity. It therefore follows that the leading behavior at the singularity, the residue, is fully accounted by the above term, and as such is gauge independent. To obtain the leading behavior we simply have to replace $V_{1}=V_{2}=0$ everywhere in the numerator in eq. (3.1). Notice that in order to reach the pole we formally need to go to complex momenta, but that is not a problem. The residue will itself be a gauge invariant function of momenta. Its continuation back to real momenta is precisely the EWA amplitude. The $1 / V_{i}$ poles in the EWA amplitude signify that the process of emission of the forward fermions and of the virtual $W$ takes place over a length and time scales much bigger than those associated with the $W W \rightarrow W W$ subprocess. It is thus intuitive that the probability for the two phenomena should factorize. On the other hand, the other diagrams and the subleading terms in eq. (3.1) do not feature such a singularity and can be interpreted as transitions where all quanta (both the fermions and the W) are emitted in a single hard collision associated to a time and length scale of order $1 / E$. The presence of a single scale $E$ implies that all the quanta are emitted in low partial waves and smoothly populate phase space: that smooth topology leads to negligible interference with the pronged topology that dominates the factorized amplitude. Notice, for instance, that when considering the Taylor expansion of the subamplitude

$$
\mathcal{A}_{\rho \sigma}\left(V_{1}^{2}, V_{2}^{2}\right)=\mathcal{A}_{\rho \sigma}(0,0)+\mathcal{A}_{\rho \sigma}^{(1,0)}(0,0) V_{1}^{2}+\mathcal{A}_{\rho \sigma}^{(0,1)}(0,0) V_{2}^{2}+\ldots
$$

the terms beyond the first, which are neglected in EWA, combine with the propagator to give non-singular contributions. These have obviously the same structure as the contributions from all the other Feynman diagrams, like figure 1b. Thus they cannot be independently gauge invariant. Indeed it was noticed long ago that, in both the unitary and $R_{\xi}$ gauges, the terms beyond the first in eq. (3.2) display an unphysical growth with powers of $E / m$. The problem of these gauges is that, for different reasons, they feature singularities at $m \rightarrow 0$. The unitary gauge is singular in both the propagator and the external polarization vectors. The $R_{\xi}$ gauge has a well behaved propagator, however there is freedom in the choice of the polarization vectors decribing the longitudinal polarizations. The usual choice, where the Goldstones fields are set to zero, is also singular. Because of these singularities, it turns out that the formally subleading terms in eq. (3.2) are instead parametrically enhanced. In some cases there is one power of $E / m$ for each external line in the $W W \rightarrow W W$ subprocess and one finds [25]

$$
\frac{\mathcal{A}^{(1,0)}(0,0)}{\mathcal{A}(0,0)} \sim \frac{1}{E^{2}}\left(\frac{E}{m}\right)^{4}
$$

implying that at $E \gg m$, the numerical value of the diagram is completely dominated by the "unphysical" gauge dependent contribution. In the gauges where that happens power counting is not manifest, and in order to establish EWA one cannot just neglect some diagrams and approximate others. In those gauges the computation of the full set of dia- 
grams is needed, in order to take into account for numerically formidable, but conceptually trivial, cancellations.

On the other hand the axial gauge, which was already adopted by KS, does not suffer from the above problems in that it is both physical and, above all, non-singular at $m \rightarrow 0$. The axial gauge is therefore the obvious choice to properly power-count all diagrams and establish the validity of EWA at the quantitative level. It is the choice we shall make in this section, by focussing on the general process $q X \rightarrow q^{\prime} Y$. In subsection 3.1 we shall describe the main technical tools, while in subsections 3.2 and 3.3 we shall expand the subamplitudes to establish EWA. In sections 3.4 and 3.5 we shall discuss the parametric dependence of the corrections to EWA.

\subsection{Axial gauge and Feynman diagrams}

As just explained, we shall work in the axial gauge. Following [28] and references therein, we fix the gauge by picking a space-like 4 -vector $n^{\mu}$ aligned along the beam direction

$$
n^{\mu}=(0,0,0,1)^{\mu} .
$$

For each of the three $Q= \pm 1,0$ charge eigenstates, the gauge-fixing condition

$$
n^{\mu} W_{\mu}^{Q}=0
$$

is strictly enforced by a delta-function in the functional integral. That ensures the field independence of the gauge variation of the gauge-fixing functional and the decoupling of the ghosts. Unlike in the covariant $R_{\xi}$ gauges, in the axial gauge the mixings among gauge fields and Goldstones are not cancelled and the propagators are thus non-diagonal. They can be regarded as matrices in the 5 -dimensional space formed by the $4 W_{\mu}$ components plus the Goldstone and are given by

$$
\mathcal{P}_{I J}=\frac{i}{q^{2}-m^{2}} \mathcal{N}_{I J},
$$

with

$$
\begin{aligned}
& \mathcal{N}_{\mu \nu}(q)=-\eta_{\mu \nu}+\frac{q_{\mu} n_{\nu}+q_{\nu} n_{\mu}}{q_{L}}+\frac{q_{\mu} q_{\nu}}{q_{L}^{2}}, \\
& \mathcal{N}_{\mu g}(q)=\mathcal{N}_{g \mu}(q)^{*}=-i m \frac{1}{q_{L}}\left(n_{\mu}+\frac{q_{\mu}}{q_{L}}\right), \\
& \mathcal{N}_{g g}(q)=1+\frac{m^{2}}{q_{L}^{2}} .
\end{aligned}
$$

The three lines of the above equations describe respectively gauge-gauge, gauge-Goldstone and Goldstone-Goldstone propagation, while $q_{L} \equiv q \cdot n$ denotes the projection of $q$ along the beam direction. Obviously, because of the gauge-fixing (3.5), the propagator matrix annihilates the 5 -vector $\left(n^{\mu}, 0\right)$ and therefore has rank 4 . Moreover, as it will become apparent from the following discussion, for on-shell momentum, $q^{2}=m^{2}$, the rank of the numerator $\mathcal{N}$ is further reduced down to 3 , corresponding to the physical polarizations of 
a massive vector. That is what makes the axial gauge "physical" and therefore best suited for our proof of EWA.

For the process $q X \rightarrow q^{\prime} Y$, considering the virtuality $V^{2} \equiv m^{2}-\left(P_{q}-P_{q}^{\prime}\right)^{2}$ defined in eq. (2.4), we can divide the Feynman diagrams into two classes. The first class, that we name "scattering diagrams", consists of the diagrams with a $1 / V^{2}$ pole (see figures 3 ), (8b), while the second class consists of all the others. In particular, among various topologies, the second class contains diagrams with $W$ 's radiated from the $q q^{\prime}$ fermion line and thus we refer to it as the class of "radiation diagrams", though the term is somewhat improper. An example of radiation diagram is shown in figure $8 \mathrm{a}$, but other examples can be obtained from a scattering diagram like figure $8 \mathrm{~b}$, by permuting $q^{\prime}$ with a quark in the inclusive final state $Y$. Notice that although the contribution of any given diagram is gauge dependent, the total contribution featuring a $1 / V$ pole is obviously gauge independent. Now, assessing the validity of the EWA amounts to assessing by how much and in which kinematic regime the $1 / V$ pole part in the scattering diagrams dominates over all the other contributions. In practice, by working in the axial gauge, it will be enough to consider just the scattering diagrams and focus on their expansion in $\delta_{\perp}$ and $\delta_{m}$ in the kinematic region where $V \ll E$. In other words, the radiation diagrams neither imply further cancellations nor bring in more sizeable corrections. That is because of two reasons. First of all, as we shall see below, in the axial gauge power counting is straightforward: unlike what happens in covariant gauges or in the unitary gauge, the amplitudes associated with individual Feynman diagrams do not feature spurious $1 / m$ singularities. ${ }^{3}$ Secondly, it is easy to see that, under the reasonable assumption that the quarks interact with the EWSB sector only via the exchange of $W$ 's, the radiation diagrams cannot be enhanced with respect to the scattering ones by simply cranking up some coupling. That is obvious for diagrams that are obtained by permutation of quarks lines in scattering diagrams. Moreover inspection of figure 8 clarifies that it is true also for diagrams with a genuine radiation structure: given one such diagram in figure $8 \mathrm{a}$ we can construct a scattering one, figure $8 \mathrm{~b}$, by attaching the building blocks $W \rightarrow Y_{i}$ on a boson line. The new diagram contains exactly the same couplings as the original one, meaning that any large coupling which is present in the radiation diagrams must unavoidably appear also in the scattering ones. Notice that the converse is not true, the simplest example is found in the higgsless SM where the strong quadrilinear coupling among longitudinal $W$ 's, which grows with the energy as $(E / v)^{2} \gg 1$, only contributes to $q W_{L} \rightarrow q^{\prime} W_{L} W_{L}$ through a scattering diagram. In that situation, the radiation type diagrams are further suppressed and it is even more justified to neglect them. In view of the above we will not need to consider the radiation diagrams explicitly any longer, their effect is either comparable or smaller than that of the subleading terms in the scattering diagrams.

\section{$3.2 \quad$ Splitting amplitudes}

Let us now consider the generic weak process $q X \rightarrow q^{\prime} Y$ defined in section 2.1, focusing on the contributions from scattering diagrams of figure 3 to the amplitude $\mathcal{A}^{\text {sc }}\left(q X \rightarrow q^{\prime} Y\right)$. We

\footnotetext{
${ }^{3}$ In those other gauges, the unphysical singular terms cancel when summing up all diagrams thus making power counting non-straightforward.
} 

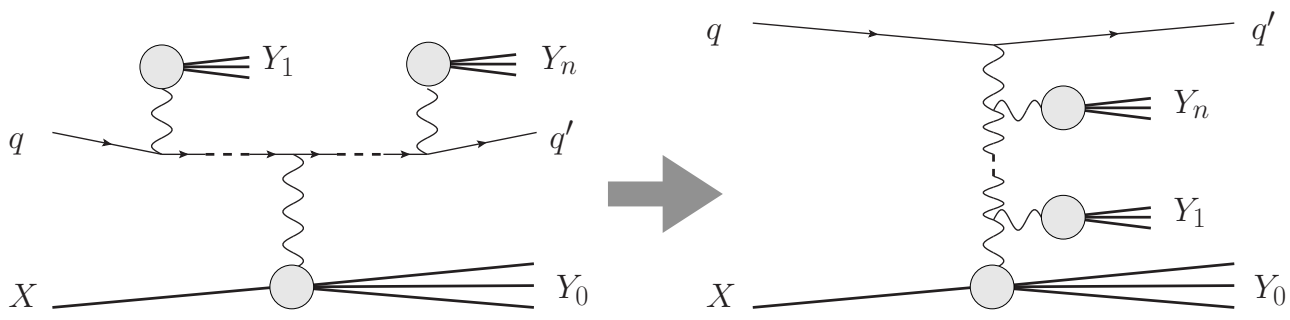

Figure 8. (a) a representative radiation diagram: unlike the scattering diagrams, these diagrams do not have the $p_{\perp} / V^{2}$ or $m / V^{2}$ singularity; (b) a representative scattering diagram: the virtual $W$ propagator leads to a $1 / V^{2}$ pole.

would like first of all to rewrite those diagrams, without any approximation, in a form suited to perform the expansion of eq. (2.2). To this end we derive a decomposition of the $\mathcal{N} \mathrm{s}$ in the vector boson propagator, for a generic off-shell momentum $q$, in terms of a suitably chosen basis of polarization vectors, by proceeding as follows. A generic 4-momentum

$$
q_{\mu}=\left(q_{0}, \vec{q}_{\perp}, q_{L}\right)_{\mu}
$$

which we take for simplicity with positive energy, but arbitrary norm $q^{2}=q_{0}^{2}-q_{L}^{2}-q_{\perp}^{2}$, can always be put in the form ${ }^{4}$

$$
\bar{q}_{\mu}=\left(\sqrt{q_{L}^{2}+q^{2}}, \overrightarrow{0}, q_{L}\right)_{\mu},
$$

by means of a Lorentz boost in a direction orthogonal to the beam. Such a boost also leaves $n^{\mu}$ invariant. One can explicitly check that the boost characterized by the velocity $\vec{\beta}=\left(\vec{q}_{\perp} / q_{0}, 0\right)$ does the job

$$
B\left(\vec{q}_{\perp} / q_{0}, 0\right)_{\mu}{ }^{\nu} \bar{q}_{\nu}=q_{\mu}, \quad B\left(\vec{q}_{\perp} / q_{0}, 0\right)_{\mu}{ }^{\nu} n_{\nu}=n_{\mu},
$$

where $B(\vec{\beta})_{\mu}{ }^{\nu}$ denotes the standard boost matrix. Given that $n$ is invariant under $B$, it is convenient to work out the basis of polarization vectors in the boosted frame where $q$ takes the form of eq. (3.9), and then obtain the ones in the original frame by boosting back with $B$. In the boosted frame the $\mathcal{N}_{\mu \nu}$ matrix takes a very simple diagonal form and it is immediate to identify the "natural" basis for its decomposition. Boosting it back we obtain

$$
\begin{aligned}
& \mathcal{N}_{\mu \nu}(q)=\sum_{h= \pm 1}\left(\varepsilon_{\mu}^{h}\right)^{*} \varepsilon_{\nu}^{h}+\frac{1+q_{L}^{2} / m^{2}}{1+q_{L}^{2} / q^{2}} \varepsilon_{\mu}^{0} \varepsilon_{\nu}^{0} \\
& \mathcal{N}_{\mu g}(q)=\varepsilon_{\mu}^{0} \varepsilon_{g} \\
& \mathcal{N}_{g g}(q)=\varepsilon_{g}^{*} \varepsilon_{g},
\end{aligned}
$$

\footnotetext{
${ }^{4}$ The discussion which follows assumes that $q_{0}^{2}-q_{\perp}^{2}=q_{L}^{2}+q^{2}>0$, this condition obviously holds for the virtual $W$ momentum of eq. (2.3) to which these manipulations will be applied.
} 
where

$$
\begin{aligned}
& \varepsilon_{\mu}^{ \pm 1}(q)=B\left(\vec{q}_{\perp} / q_{0}, 0\right)_{\mu}{ }^{\nu}(0,1 / \sqrt{2}, \mp i / \sqrt{2}, 0)_{\nu} \\
& \varepsilon_{\mu}^{0}(q)=B\left(\vec{q}_{\perp} / q_{0}, 0\right)_{\mu}{ }^{\nu}\left(-\frac{\sqrt{1+q^{2} / q_{L}^{2}}}{\sqrt{1+q_{L}^{2} / m^{2}}}, 0,0,0\right)_{\nu}=-\frac{m}{q_{L} \sqrt{1+m^{2} / q_{L}^{2}}}\left(n_{\mu}+\frac{q_{\mu}}{q_{L}}\right), \\
& \varepsilon_{g}(q)=i \sqrt{1+m^{2} / q_{L}^{2}} .
\end{aligned}
$$

Clearly, the above equations rely on a choice of the normalizations and on the definition of the Goldstone's wave function $\varepsilon_{g}$, which we performed in such a way to get unit coefficient in the decomposition (3.10) of $\mathcal{N}_{\mu g}$, of $\mathcal{N}_{g g}$ and of the transverse part of $\mathcal{N}_{\mu \nu}$.

In the on-shell limit $q^{2} \rightarrow m^{2}$ the numerator $\mathcal{N}_{I J}$ reduces, as anticipated, to a rank-3 matrix and its 3 non-null eigenvectors span the space of the physical $W$ states. Following [28], we choose as a basis the two "transverse" wave-functions given by the 5-vectors $E_{I}^{ \pm 1}=\left(\varepsilon^{\mu \pm 1}, 0\right)$, plus the "longitudinal" one $E_{I}^{0}=\left(\varepsilon^{\mu 0}, \varepsilon_{g}\right)$, and $\mathcal{N}_{I J}=\sum_{h= \pm, 0}\left(E_{I}^{h}\right)^{*} E_{J}^{h}$. Notice that with this definition the polarized $W$ states are not exactly eigenstates of the helicity but of the angular momentum along the beam direction in the $q=\bar{q}$ frame as it becomes apparent from eq. (3.11) by checking that the polarization states transform with a phase under rotations in the plane transverse to the beam. In the lab frame, the transformation property of the polarization vectors under transverse rotations is also simple, let us work it out for future use. Consider a rotation $R_{\mu}{ }^{\nu}(\theta)$ of the momentum $q$ in the transverse plane, defined by

$$
q_{\mu}^{\prime} \rightarrow q_{\mu}^{(\theta)}=R_{\mu}^{\nu}(\theta) q_{\nu}=\left(q_{0}, \cos \theta q_{\perp}^{1}+\sin \theta q_{\perp}^{2}, \cos \theta q_{\perp}^{2}-\sin \theta q_{\perp}^{1}, q_{L}\right)_{\mu}
$$

The composition rules of Lorentz transformations imply that

$$
B\left(\vec{q}_{\perp}^{(\theta)} / q_{0}, 0\right)_{\mu}{ }^{\nu}=R_{\mu}{ }^{\mu^{\prime}} B\left(\vec{q}_{\perp} / q_{0}, 0\right)_{\mu^{\prime}}^{\nu^{\prime}} R^{-1} \underset{\nu^{\prime}}{\nu}
$$

from which it is immediate to check that the polarization vectors (3.11) satisfy

$$
R_{\mu}^{-1}{ }^{\nu} \varepsilon_{\nu}^{h}\left(q^{(\theta)}\right)=e^{i h \theta} \varepsilon_{\mu}^{h}(q),
$$

with $h= \pm 1,0$. According to the above equation, which is valid for a generic off-shell momentum $q$, our polarization vectors correspond to wave functions with (total) angular momentum $h$ along the beam axis, $J_{3}=h$.

Let us now consider a generic scattering diagram, we will rewrite its amplitude by applying the decomposition (3.10). Notice that in the axial gauge, as figure 3 shows, there are two kind of scattering diagrams, which we denote as type "A" and "B" depending on whether the fermion line is attached to a gauge-gauge or to a gauge-Goldstone propagator. There is no contribution from the Goldstone-Goldstone propagator because massless quarks do not couple directly to the Goldstones. The momentum $K$ flowing in the $W$ propagator is given by eq. (2.3), using eq. (3.10) we rewrite each scattering diagram, up to 
corrections of $\mathcal{O}\left(\delta_{\perp}^{2}\right)+\mathcal{O}\left(\delta_{m}^{2}\right)$ that originate from expanding the second term in the first line of eq. (3.10), as

$$
\begin{aligned}
\mathcal{A}^{\text {sc. }-A}= & -\frac{i}{V^{2}} \sum_{h= \pm 1}\left[J^{\mu}\left(\varepsilon_{\mu}^{h}\right)^{*}\right]\left[\varepsilon_{\nu}^{h} \mathcal{A}_{Q}^{\nu}\right] \\
& -\frac{i}{V^{2}}\left[J^{\mu}\left(\varepsilon_{\mu}^{0}\right)^{*}\right]\left[\left(1-\frac{V^{2}}{m^{2}}\right) \varepsilon_{\nu}^{0} \mathcal{A}_{Q}^{\nu}\right]\left[1+\mathcal{O}\left(\delta_{\perp}^{2}+\delta_{m}^{2}\right)\right], \\
\mathcal{A}^{\text {sc. }-B}= & -\frac{i}{V^{2}}\left[J^{\mu}\left(\varepsilon_{\mu}^{0}\right)^{*}\right]\left[\varepsilon_{s} \mathcal{A}_{Q}^{g}\right],
\end{aligned}
$$

where $\mathcal{A}_{Q}^{\nu}$ denotes the "hard" amputated amplitude of the gauge field $W_{Q}^{\nu}$ on the $X$ and $Y$ states, while $\mathcal{A}_{Q}^{g}$ is the Goldstone amplitude on the same external states. The "soft" part of the scattering diagrams, associated to the quark vertex in eq. (2.1), is instead contained in the chiral current $J$, which is given by

$$
J^{\mu}\left(P_{q^{\prime}}, P_{q}\right) \equiv-2 i C \bar{u}_{L}\left(P_{q^{\prime}}\right) \gamma^{\mu} u_{L}\left(P_{q}\right)=-2 i C \chi^{\dagger}\left(P_{q^{\prime}}\right) \bar{\sigma}^{\mu} \chi\left(P_{q}\right),
$$

where $C$ depends on the charge of the virtual $W$ and is given in eq. (2.8). The equation above has been written in the Weyl basis where the $\gamma$ matrices are

$$
\gamma^{\mu}=\left(\begin{array}{cc}
0 & \sigma^{\mu} \\
\bar{\sigma}^{\mu} & 0
\end{array}\right), \quad \gamma^{5}=\left(\begin{array}{cc}
-\mathbb{1} & 0 \\
0 & \mathbb{1}
\end{array}\right),
$$

and the wave function for massless spinors of helicity $-1 / 2$ and generic momentum $P_{\mu}=$ $\left(p_{0}, p_{1}, p_{2}, p_{3}\right)_{\mu}$ can be taken to be

$$
u_{L}(P)=\left(\begin{array}{c}
\chi(P) \\
0
\end{array}\right), \quad \chi(P)=\frac{1}{\sqrt{p_{0}+p_{3}}}\left(\begin{array}{c}
p_{0}+p_{3} \\
p_{1}+i p_{2}
\end{array}\right) .
$$

The above choice for the spinor wave function is fully analogous to the choice of polarization vectors in eq. (3.13): it is obtained by boosting the helicity eigenfunction $\chi_{0}$ from the frame where $p_{\perp}=0$ to the lab frame

$$
\chi(P)=\Lambda_{L} \chi_{0}=\Lambda_{L}\left(p_{\perp} / p_{0}\right)\left(\begin{array}{c}
\sqrt{2 p_{3}} \\
0
\end{array}\right)
$$

where

$$
\Lambda_{L}\left(p_{\perp} / p_{0}\right)=e^{-\eta_{i} \sigma_{i} / 2} \quad \eta_{i}=\frac{p_{\perp}^{i}}{2\left|p_{\perp}\right|} \ln \left(\frac{1-\left|p_{\perp}\right| / p_{0}}{1+\left|p_{\perp}\right| / p_{0}}\right) .
$$

In analogy with eq. (3.13), the spinor wave functions transform under rotations in the transverse plane according to

$$
e^{-i \theta \sigma_{3} / 2} \chi\left(p^{(\theta)}\right)=e^{-i \theta / 2} \chi(p)
$$

and thus correspond to eigenstates with $J_{3}=-1 / 2$. A consequence of the above is that the current, being helicity preserving, transforms as a vector under rotations in the transverse plane, without the appearance of extra momentum-dependent phases.

$$
J^{\mu}\left(P^{\prime(\theta)}, P^{(\theta)}\right)=R_{\mu^{\prime}}^{\mu} J^{\mu^{\prime}}\left(P^{\prime}, P\right) .
$$


It is a simple exercise, at this point, to compute the "splitting amplitudes", i.e. the soft part of the amplitude (3.14). In the expansion of eq. (2.2) the result is

$$
\begin{aligned}
-\frac{i}{V^{2}}\left[J^{\mu}\left(\varepsilon_{\mu}^{ \pm 1}\right)^{*}\right] & =2 C \frac{p_{\perp} e^{ \pm i \phi}}{V^{2}} g_{ \pm}(x)\left[1+\mathcal{O}\left(\delta_{\perp}^{2}+\delta_{m}^{2}\right)\right], \\
-\frac{i}{V^{2}}\left[J^{\mu}\left(\varepsilon_{\mu}^{0}\right)^{*}\right] & =2 C \frac{m}{V^{2}} g_{0}(x)\left[1+\mathcal{O}\left(\delta_{\perp}^{2}+\delta_{m}^{2}\right)\right],
\end{aligned}
$$

where

$$
\begin{aligned}
& g_{+}(x)=\sqrt{2} \frac{\sqrt{1-x}}{x}, \\
& g_{-}(x)=\sqrt{2} \frac{1}{x \sqrt{1-x}}, \\
& g_{0}(x)=2 \frac{\sqrt{1-x}}{x} .
\end{aligned}
$$

The dependence of the result on

$$
p_{\perp} e^{-i \phi} \equiv p_{\perp}^{1}-i p_{\perp}^{2} \equiv \tilde{p}_{\perp}
$$

follows from eqs. (3.13) and (3.21), according to which the splitting amplitude into a vector boson of helicity $h$ changes by a phase $e^{-i h \theta}$ under a rotation in the transverse plane. That result is a reflection of angular momentum conservation: the amplitude for helicity $h$ corresponds to a final state with orbital angular momentum $-h$ so as to compensate for the spin of the vector boson. By the resulting selection rule the longitudinal splitting amplitude arises at zeroth order in $p_{\perp}$ while the transverse splitting amplitudes only arise at first order. Similarly the subleading corrections to all amplitudes are quadratic in $p_{\perp}$. Notice that the subleading corrections are also quadratic in the mass $m$, because $m$ appears linearly only in the prefactor of the longitudinal splitting amplitude while all the other terms are quadratic. That result is associated to a reparametrization of the lagrangian and the fields under which the gauge fields are invariant, $W_{\mu} \rightarrow W_{\mu}$, while the Goldstone fields are odd, $\pi \rightarrow-\pi$, and the mass changes sign, $m \rightarrow-m$ (see discussion in section 3.4).

\subsection{Derivation of EWA}

In the previous section we worked out the fermion current factors in eq. (3.14), which are associated with the collinear splitting $q \rightarrow q^{\prime} W$. Next we should deal with the factors associated with the hard transition. Those we would like to replace with the on-shell $W X \rightarrow Y$ scattering amplitudes. The hard amplitudes $\mathcal{A}_{Q}^{\nu, g}$ and the polarization vectors $\varepsilon^{h, g}$ depend on the momentum of the virtual $W$, parametrized by $K$ in eq. (2.3). As $K$ is nearly on shell it is convenient to rewrite it as

$$
K=\left(\omega_{\vec{k}} \sqrt{1-V^{2} / \omega_{\vec{k}}^{2}}, \vec{k}\right),
$$

where $\omega_{\vec{k}}=\sqrt{m^{2}+|\vec{k}|^{2}}$ is the on-shell energy of a real $W$ with the same 3 -momentum $\vec{k}=\left(-\vec{p}_{\perp}, x E\right)$ as the virtual $W$. The deviations from on-shellness only affect the energy 
component $K^{0}$ of $K$ by an amount of relative size $\mathcal{O}\left(V^{2} / \omega_{\vec{k}}^{2}\right)=\mathcal{O}\left(\delta_{\perp}^{2}+\delta_{m}^{2}\right)$. In the hard region the amplitudes are well behaved functions of the external momenta and so are the wave functions. Thus the subprocess amplitudes in eq. (3.14) can be safely expanded in the virtuality $V$

$$
[\epsilon \cdot \mathcal{A}] \equiv \mathcal{M}\left(V^{2}\right)=\mathcal{M}(0)+\mathcal{M}^{\prime}(0) V^{2}+O\left(V^{4}\right) .
$$

The reasonable expectation is that, barring cancellations that we shall discuss below, the radius of convergence of the above expansion is controlled by the hardness of the subprocess, as quantified by the virtuality $H$ of its internal lines, that is $\mathcal{M}^{\prime}(0) / \mathcal{M}(0) \sim 1 / H^{2}$. By assuming, for simplicity, $H \sim E$, we can then write

$$
\begin{aligned}
\mathcal{A}^{\text {sc. }-A}= & 2 C \frac{p_{\perp}}{V^{2}} \sum_{h= \pm 1} e^{\mp i \phi} g_{ \pm}(x)\left[\varepsilon_{\nu}^{+} \mathcal{A}^{\nu}\right]_{\mathrm{on}}(\vec{k})\left[1+\mathcal{O}\left(\delta_{\perp}^{2}+\delta_{m}^{2}\right)\right] \\
& +2 C \frac{m}{V^{2}} g_{0}(x)\left[\varepsilon_{\nu}^{0} \mathcal{A}^{\nu}\right]_{\mathrm{on}}(\vec{k})\left[1+\mathcal{O}\left(\delta_{\perp}^{2}+\delta_{m}^{2}\right)\right] \\
& +2 C \frac{1}{k_{L}} g_{0}(x) \mathcal{A}_{\text {local }}, \\
\mathcal{A}^{\text {sc. }-B}= & 2 C \frac{m}{V^{2}} g_{0}(x)\left[\varepsilon_{g} \mathcal{A}^{g}\right]_{\mathrm{on}}(\vec{k})\left[1+\mathcal{O}\left(\delta_{\perp}^{2}+\delta_{m}^{2}\right)\right]
\end{aligned}
$$

where all the amplitudes and the polarization vectors in the square brackets are computed, as explicitly indicated, for an on-shell $W$ with 4 -momentum $\left(\omega_{\vec{k}}, \vec{k}\right)$. The term denoted as $\mathcal{A}^{\text {local }}$, collects all the contributions proportional to the $V^{2} / \mathrm{m}^{2}$ term in the parenthesis in the second line of eq. (3.14), and is thus non singular as $V \rightarrow 0$. Now, focusing on the interesting regime $V \sim m \sim p_{\perp}$, it is instructive to power count the various terms. Those associated with the on-shell amplitudes are $O(1 / V)$. The presence of a $1 / V$ pole indicates that the virtual $\mathrm{W}$ is emitted and absorbed at well separated spacetime points. On the other hand the term $\mathcal{A}^{\text {local }}$ is $O\left(V^{0}\right)$ and not-singular as $V \rightarrow 0$, like the local amplitude mediated by a contact term. Thus the term $\mathcal{A}^{\text {local }}$ belongs to the same class as the contribution from the diagrams with radiation topology described in section 3.1. Explicitly we find

$$
\mathcal{A}^{\text {local }}=\left(n_{\nu}+K_{\nu} / k_{L}\right) \mathcal{A}_{Q}^{\nu}\left[1+\mathcal{O}\left(\delta_{\perp}^{2}+\delta_{m}^{2}\right)\right],
$$

where we made use of the explicit form of the $\varepsilon^{0}$ vector in eq. (3.11). Finally, notice that the subleading corrections to the $1 / V$ pole terms, those associated with $\delta_{\perp}^{2}$ and $\delta_{m}^{2}$, correspond to $O(V)$ contributions, that are thus further suppressed with respect to $\mathcal{A}^{\text {local }}$.

The above discussion would be invalidated by the occurrence of on-shell cancellations, that badly change our estimate $\mathcal{M}^{\prime}(0) / \mathcal{M}(0) \sim 1 / H^{2}$. The most obvious thing that can happen is that $\mathcal{M}(0)=0$, corresponding to a vanishing amplitude for the subprocess, which is not our hypothesis. ${ }^{5}$ Leaving out the trivial case $\mathcal{M}(0)=0$, we can identify two other more subtle sources for such cancellations, one physical and one unphysical. The first possibility, corresponding to a genuine physical effects, is that "helicity selection rules" ${ }^{6}$ suppress $\mathcal{M}(0)$ by some power of $m / E$ with respect to naive power counting. This

\footnotetext{
${ }^{5}$ For $\mathcal{M}(0)=0$ the $1 / V$ pole is absent, corresponding to the possibility of parametrizing the $q X \rightarrow q^{\prime} Y$ process by a contact interaction. That can be done via a field redefinition or, equivalently, by using the $W$ equations of motion.

${ }^{6}$ Of the type involved in the cancellation of MHV amplitudes.
} 
could happen for peculiar polarizations of the external states described by $X$ and $Y$, but we do not expect it to happen for all polarizations. Thus for sufficiently inclusive initial and final states there should be no issue: the estimate of the corrections in eq. (3.27) will safely apply to the dominant helicity channels, which of course are the ones which are not subject to cancellations. In section 3.5 we will discuss examples of these these helicity induced cancellations. The second possibility, associated instead to an unphysical effect, arises in gauges where the propagator and/or the polarization vectors are singular for $m \rightarrow 0$. In those gauges, that include the unitary and $R_{\xi}$ gauges but not the axial gauge, naive power counting is spoiled by the presence of terms which, diagram by diagram, grow off-shell like powers of $E / m$. More precisely, in those gauges one finds spurious contributions $\mathcal{M}^{\prime}(0) / \mathcal{M}(0) \sim E^{2} / m^{4}$ that completely invalidate an approach based on diagrammatics [25]. Those spurious terms are unphysical and cancel only upon summation of the whole set of diagrams. The axial gauge has the great advantage that the cancellation is manifest diagram by diagram, simply because no such term can arise due to the absence of $1 / m$ singularities! The origin of this problem is simply that $\mathcal{M}^{\prime}(0)$, unlike $\mathcal{M}(0)$, is an unphysical gauge dependent quantity. Because of the above issue, the validity of EWA was put in doubt by ref. [26, 27]. Our basic point, that simply follows and extends the discussion in [28], is that the problem is easily avoided by working in the axial gauge. In fact EWA can also conveniently be derived by working in the $R_{\xi}$ gauge, by noticing that one has the freedom of chosing a parametrization where the polarization vectors are non-singular as $m \rightarrow 0$. That is the parametrization that is most suitable for proving the equivalence between longitudinally polarized vectors and eaten Goldstones in high-energy scattering. We will present this alternative approach in a forthcoming paper.

In eq. (3.27) we can already recognize the various terms of the EWA formula reported in eq. (2.5). At the leading order, the terms in the first line in $\mathcal{A}^{s c .-A}$ coincide with the $W_{Q} X \rightarrow Y$ scattering amplitude with transversely polarized incoming $W$ 's while the term in second line, in combination with $\mathcal{A}^{\text {sc. }-B}$, reconstructs the longitudinally polarized amplitude. Indeed, in the axial gauge, the on-shell $W_{L}$ state is a coherent superposition of the gauge and Goldstone fields. That is why the amplitude for $W_{L}$ involves the sum of two terms. Using a compact notation the total $q X \rightarrow q^{\prime} Y$ amplitude can therefore be rewritten as

$$
\begin{aligned}
\mathcal{A} & =\frac{2 C}{V^{2}}\left[\tilde{p}_{\perp} \mathcal{A}_{+}+\tilde{p}_{\perp}^{*} \mathcal{A}_{-}+m \mathcal{A}_{0}+\frac{V^{2}}{k_{L}} \mathcal{A}_{\text {local }}\right]\left[1+\mathcal{O}\left(\delta_{m}^{2}+\delta_{\perp}^{2}\right)\right] \\
\mathcal{A}_{ \pm} & =g_{ \pm}(x)\left[\varepsilon_{\nu}^{ \pm} \mathcal{A}^{\nu}\right]_{\text {on }}(\vec{k}), \quad \mathcal{A}_{0}=g_{0}(x)\left\{\left[\varepsilon_{\nu}^{0} \mathcal{A}_{Q}^{\nu}\right]_{\mathrm{on}}(\vec{k})+\left[\varepsilon_{g} \mathcal{A}^{g}\right]_{\mathrm{on}}(\vec{k})\right\} .
\end{aligned}
$$

Eq. (3.29), by neglecting the subleading $\mathcal{A}_{\text {local }}$ term (as well as the $\delta_{\perp}^{2}$ and $\delta_{m}^{2}$ corrections) yelds a "generalized EWA"

$$
\mathcal{A}_{\mathrm{gEWA}}=\frac{2 C}{V^{2}}\left[\tilde{p}_{\perp} \mathcal{A}_{+}+\tilde{p}_{\perp}^{*} \mathcal{A}_{-}+m \mathcal{A}_{0}\right]
$$

which provides an approximation for the amplitude of the complete process in terms of the hard on-shell scattering amplitude of an equivalent $W$ boson. The resulting differential cross section is more accurate than the standard approximation in eq. (2.5), because it 
maintains the information on the $\phi$ distribution of the forward jet, which is instead integrated in the standard EWA. The $\phi$ distribution is determined by the interference among the individual subamplitudes in eq. (3.31). Moreover, as we shall clarify better below, for processes dominated by $\mathcal{A}_{0}$, as it often happens at sufficiently high energy in composite Higgs models, this generalized EWA includes in a consistent manner the leading $O\left(\delta_{\perp}\right)$ corrections to the differential rate coming from the interference between $\mathcal{A}_{0}$ and $\mathcal{A}_{ \pm}$. We will discuss the implications of this generalized EWA formula in a forthcoming paper.

To obtain eq. (2.5) we just need to further expand the subamplitudes in $\frac{\tilde{p}_{\perp}}{E}, \frac{\tilde{p}_{\perp}^{*}}{E}$

$$
\mathcal{A}_{i}=\mathcal{A}_{i}^{(0,0)}+\mathcal{A}_{i}^{(1,0)} \frac{\tilde{p}_{\perp}}{E}+\mathcal{A}_{i}^{(0,1)} \frac{\tilde{p}_{\perp}^{*}}{E} \ldots
$$

for $i=(+,-, 0$, local $)$ and keep only the very leading term

$$
\mathcal{A}^{(0,0)}=\frac{2 C}{V^{2}}\left[\tilde{p}_{\perp} \mathcal{A}_{+}^{(0,0)}+\tilde{p}_{\perp}^{*} \mathcal{A}_{-}^{(0,0)}+m \mathcal{A}_{0}^{(0,0)}\right]
$$

The resulting approximation corresponds to replacing the momentum $\vec{k}=\left(-\vec{p}_{\perp}, x E\right)$ of the equivalent $W$, with a fully collinear one $\vec{k}_{W}=(-\overrightarrow{0}, x E)$ as in eq. (2.6). Modulo the splitting function factor in front (see eq. (3.30)), $A_{+,-, 0}^{(0,0)}$ represent the $W X \rightarrow Y$ amplitudes for a fully collinear equivalent $W$. We thus find the differential cross section

$$
\begin{aligned}
& d \sigma\left(q X \rightarrow q^{\prime} Y\right)_{\mathrm{EWA}} \\
&=\frac{1}{2 E_{q} 2 E_{X}\left|1-v_{X}\right|} \int_{\phi} \frac{\left|\mathcal{A}^{(0,0)}\right|^{2}}{2} \frac{d^{3} P_{q^{\prime}}}{2 E_{q^{\prime}}(2 \pi)^{3}} d \Phi_{Y}(2 \pi)^{4} \delta^{4}\left(P_{Y}^{\mathrm{tot}}+P_{q^{\prime}}-P_{q}-P_{X}\right) \\
& \simeq \frac{2 C^{2}}{V^{4}} \cdot \frac{p_{\perp} d p_{\perp} x d x}{(2 \pi)^{2} 2(1-x)} \times\left[p_{\perp}^{2}\left|\mathcal{A}_{+}^{(0,0)}\right|^{2}+p_{\perp}^{2}\left|\mathcal{A}_{-}^{(0,0)}\right|^{2}+m^{2}\left|\mathcal{A}_{0}^{(0,0)}\right|^{2}\right] \\
& \times \frac{1}{2 E_{q} 2 E_{W}\left|v_{W}-v_{X}\right|} d \Phi_{Y}(2 \pi)^{4} \delta^{4}\left(P_{Y}^{\mathrm{tot}}-K_{W}-P_{X}\right)
\end{aligned}
$$

where $d \Phi_{Y}$ denotes the phase space of the final state $Y$ and the $1 / 2$ factor comes from the average on the two polarizations of the incoming $q$. Notice that by performing the $d \phi$ integral the interference terms cancel, since the $\mathcal{A}_{i}^{(0,0)}$ do not depend on $\phi$. To obtain the second equality we have employed a few kinematic relations that are easily extracted from section 2.1, in particular we used that $E_{q}=E, d^{3} P_{q^{\prime}}=E p_{\perp} d p_{\perp} d \phi d x$ and that $E_{W}=x E$, $E_{q^{\prime}}=(1-x) E$ up to quadratic corrections, we also used $v_{W} \simeq 1$ in the relative velocity term. The incoherent sum of squared amplitudes, when taking into account the splitting function coefficient in their definition eq. (3.30), together with the flux and phase space factor for the $W X \rightarrow Y$ process in the third line, are then easily seen to reproduce eq. (2.5).

\subsection{Corrections to EWA}

In the previous section we have established the validity of EWA in the formal limit of extremely high energy, much above the jet $p_{\perp}$ and the $W$ mass. This result is reassuring as it guarantees the observability in principle (i.e., under ideal experimental conditions) of the on-shell $W$ boson collisions. In practice, however, the energy is limited and the EWA formula could receive large corrections. A hypothetical measurement of the equivalent $W$ 
boson scattering could therefore be affected by a potentially large intrinsic (systematic) error which is very important to quantify. This is the aim of the present section, in which we derive a parametric estimate of the deviations of the exact cross section from the EWA formula.

To start with, let us discuss the corrections due to the subleading terms in equation (3.30), which we neglected in order to obtain the generalized EWA formula of eq. (3.31). Aside from the generic $\delta_{\perp}^{2}, \delta_{m}^{2}$ that originate from the expansion of the various matrix elements, corrections arise from the $\mathcal{A}_{\text {local }}$ term, which is seemingly suppressed only by one power of $\delta_{\perp}, \delta_{m}$ and therefore potentially gives the most sizable effect. However the relevance of $\mathcal{A}_{\text {local }}$ crucially depends on the relative size of the amplitudes for different helicities $\mathcal{A}_{ \pm}, \mathcal{A}_{0}, \mathcal{A}_{\text {local }}$. In general different sizes for the amplitudes involving the gauge fields on one side and the Goldstone bosons on the other are expected. That is particularly true in models where the electroweak symmetry breaking sector is strongly coupled. Thus, while we expect $\mathcal{A}_{ \pm} \sim \mathcal{A}_{\text {local }}$ as they both just involve the gauge field on the equivalent $W$ line, it turns out that $\mathcal{A}_{0}$ and $\mathcal{A}_{ \pm}$typically have rather different sizes. In order to understand this point let us recall a useful selection rule controlling the appearance of powers of $m$ in physical quantities. The lagrangian for Goldstones $\pi$ and gauge fields $W_{\mu}$ (with or without a SM Higgs) is invariant under the reparametrization

$$
W_{\mu} \rightarrow W_{\mu}, \quad \pi \rightarrow-\pi, \quad m \rightarrow-m,
$$

according to which the sign of $m$ is not a physical observable. Since the probability $\mathcal{A A}^{*}$ is an observable, the amplitude $\mathcal{A}$ must be either even or odd under $m \rightarrow-m$. Indeed, because of eq. (3.35) and because of the structure of the polarization vectors $E_{I}^{ \pm}, E_{I}^{0}$ (below eq. (3.11)), we conclude that $\mathcal{A}_{ \pm}$and $\mathcal{A}_{0}$ have opposite parities (more directly one can deduce that by noticing the relative power of $m$ with which they enter $\mathcal{A}$ ). In full generality we must then have

$$
\frac{\mathcal{A}_{ \pm}}{\mathcal{A}_{0}} \sim\left(\frac{m}{E}\right)^{2 n+1} b,
$$

where $b$ is a dimensionless ratio of couplings. In most cases (as in the examples of the following section) the simplest possibility is realized, that is either $n=0$ or $n=-1$, so that the asymptotically subleading polarized amplitude is suppressed with respect to the leading one by $m / E$. In practice we therefore need to consider only two cases:

$$
\text { 1) } \frac{\left|\mathcal{A}_{ \pm}\right|}{\left|\mathcal{A}_{0}\right|} \sim \frac{m}{E} b, \quad \text { 2) } \frac{\left|\mathcal{A}_{0}\right|}{\left|\mathcal{A}_{ \pm}\right|} \sim \frac{m}{E} b,
$$

(we stress that since the two cases are logically distinct, the coefficient $b$ has different meaning and size in the two cases). From the above equations we see that in the typical situation there will be a hierarchy, due to the $m / E$ factor, among $\mathcal{A}_{ \pm}$and $\mathcal{A}_{0}$. It is however possible to obtain $\left|\mathcal{A}_{ \pm} / \mathcal{A}_{0}\right|=O(1)$, but only in a specific range of energies and by the compensating effect of a very large $b$, of order $E / m$. We will illustrate an example of that in the following section, notice however that the by far more common situation is $b=O(1)$. 
In order to better understand the nature of the $b$ parameter it is useful to restore the dimensionality of $\hbar$. That way the coupling associated to an $n$-field vertex has dimensionality $n / 2-1$. For instance the gauge coupling $g$ and the Higgs quartic $\lambda$ have respectively dimension $1 / 2$ and 1 . Since $b$ describes the ratio among homogeneous quantities it should be dimensionless. In any given theory we then know what powers to expect in $b$. For instance in the SM we must have $b=\left(g^{2} / \lambda\right)^{p}=\left(m^{2} / m_{H}^{2}\right)^{p}$.

Let us consider now the squared amplitude integrated over $\phi$, which is the object we need in order to derive the final EWA formula in eq. (2.5). By expanding the subamplitudes in $p_{\perp}$, as in eq. (3.32), under the reasonable assumption $E \partial_{p_{\perp}} \mathcal{A}_{i} \sim O\left(\mathcal{A}_{i}\right)$, the result has the structure

$$
\int_{\phi}|\mathcal{A}|^{2} \propto p_{\perp}^{2} \mathcal{A}_{ \pm}^{2}+m^{2} \mathcal{A}_{0}^{2}+p_{\perp}^{2} \frac{m}{E} \mathcal{A}_{ \pm} \mathcal{A}_{0}+V^{2} \frac{m}{E} \mathcal{A}_{ \pm} \mathcal{A}_{0}+\frac{V^{4}}{E^{2}} \mathcal{A}_{ \pm}^{2}+\ldots
$$

where to simplify the notation we simply indicated by $\mathcal{A}_{ \pm}$and $\mathcal{A}_{0}$ the leading terms $\mathcal{A}_{ \pm}^{(0,0)}$ and $\mathcal{A}_{0}^{(0,0)}$, defined in eq.s (3.32), (3.33), and estimated the subleading terms according to $\mathcal{A}_{i}^{(m, n)} \sim \mathcal{A}_{i}$. In eq. (3.38), the third term arises from cross terms of the type $\left(\mathcal{A}_{0}^{(0,0)}\right)^{*} \mathcal{A}_{+}^{(0,1)}$ and $\left(\mathcal{A}_{0}^{(1,0)}\right)^{*} \mathcal{A}_{+}^{(0,0)}$, while the fourth term comes from $\left(\mathcal{A}_{0}^{(0,0)}\right)^{*} \mathcal{A}_{\text {local }}^{(0,0)}$. The dots represent terms of even higher order. Notice that the leading interference between $\mathcal{A}_{ \pm}$and $\mathcal{A}_{\text {local }}$ vanishes upon integration over $\phi$. Focussing again on the physically interesting region $p_{\perp} \sim m$ we find that the relative corrections to the EWA formula scale like

$$
\delta_{\mathrm{EWA}} \sim \frac{m}{E} \frac{\left|\mathcal{A}_{ \pm} \mathcal{A}_{0}\right|}{\max \left(\left|\mathcal{A}_{ \pm}\right|^{2},\left|\mathcal{A}_{0}\right|^{2}\right)} \sim \frac{m}{E} \min \left(\left|\mathcal{A}_{0} / \mathcal{A}_{ \pm}\right|,\left|\mathcal{A}_{ \pm} / \mathcal{A}_{0}\right|\right) .
$$

We see that $\delta_{\mathrm{EWA}}$ is always smaller than $m / E$, and it become of order $m / E$ only if $\mathcal{A}_{0}$ and $\mathcal{A}_{ \pm}$are comparable.

In both cases considered in eq. (3.37) $\delta_{\mathrm{EWA}}$ becomes

$$
\delta_{\mathrm{EWA}} \sim \frac{m}{E} \min \left[\frac{m b}{E}, \frac{E}{m b}\right] .
$$

Let us analyze this formula in some more detail. For asymptotically high energies, $\delta_{\text {EWA }}$ does scale quadratically with the energy, but with a possibly large prefactor $b: \delta_{\mathrm{EWA}} \sim$ $b m^{2} / E^{2}$. The corrections grow as $E$ decreases and at the critical value $E \simeq m b$ they become of order $m / E \sim 1 / b$. This behavior could be captured by a phenomenological formula

$$
\delta_{\mathrm{EWA}}=C_{1} \frac{m^{2}}{E^{2}} b \frac{1}{1+C_{2} \frac{m^{2}}{E^{2}} b^{2}},
$$

with $C_{1,2}$ order one parameters. Notice that in the above discussion we have been implicitly assuming $b>1$. Only for that case, does eq. (3.41) represent the leading correction. Otherwise, $\delta_{\mathrm{EWA}}$ is dominated by $\delta_{\perp}^{2}, \delta_{m}^{2}$ in equation (3.30), that is the irreducible effects of taking the on-shell limit of the various matrix elements. In the end we always have $\delta_{\text {EWA }}>m^{2} / E^{2}$.

One region worth considering is $p_{\perp} \ll m$, focussing for simplicity on the more common case $b \simeq 1$. In that case, the corrections scale differently for processes dominated by the 
longitudinal or the transverse amplitude, which correspond respectively to case 1) and case 2 ) in eq. (3.37). In case 1), eq. (3.38) implies as usual

$$
\delta_{\mathrm{EWA}} \sim \frac{m^{2}}{E^{2}} .
$$

In case 2), instead, the corrections from the local term are enhanced

$$
\delta_{\mathrm{EWA}} \sim \frac{m^{2}}{E^{2}} \frac{m^{2}}{p_{\perp}^{2}}
$$

notice in particular that the EWA breaks down completely for $p_{\perp} \sim m^{2} / E$. For smaller $p_{T}$, the transverse contribution becomes negligible, while the contribution from $\mathcal{A}_{0} \sim(m / E) \mathcal{A}_{ \pm}$ scales precisely like the local contribution. Notice that a similar phenomenon does not take place for the splitting into massless vector bosons, like gluons and photons, since in that case the virtuality $V^{2}$ also goes to zero like $p_{\perp}^{2}$.

Finally, we should also consider the case of intermediate jet transverse momentum: $E \gg p_{\perp} \gg m$, in which the corrections are always

$$
\delta_{\mathrm{EWA}} \sim \frac{p_{\perp}^{2}}{E^{2}} .
$$

Notice that in the more typical situation, $b \sim 1$ and $p_{\perp} \nless m$, the corrections are simply given, as anticipated in section 2.1 , by eq. (2.10).

\subsection{The example of WW scattering}

The general considerations of the previous sections are conveniently illustrated in the explicit example of the $W W$ scattering process, which we already considered in section 2.2. We work in the Higgs model (with vanishing hypercharge, compatibly with eq. (2.1)) and we compute the totally polarized on-shell scattering amplitude $W_{p_{1}}^{+} W_{p_{2}}^{-} \rightarrow W_{p_{3}}^{+} W_{p_{4}}^{-}$. For instance, in the high energy $\left(E \gg m, E \gg m_{H}\right)$ and fixed angle limit, the ++++ amplitude (i.e., $\left\{p_{1}, p_{2}, p_{3}, p_{4}\right\}=\{1,1,1,1\}$ ), reads

$$
\mathcal{A}_{+}=-\frac{2 g^{2}}{\sin ^{2}(\theta / 2)}+\mathcal{O}\left(m^{2} / E^{2}\right),
$$

where $E$ and $\theta$ denote respectively the energy and the scattering angle of the $W^{+}$in the center of mass frame. The above result complies perfectly with the expectations of power counting for the scattering amplitude among transversely polarized $W$ 's: two powers of $g$ and constant scaling with energy. Moreover the Coulomb singularity at $\theta=0$ is due to the negligibility of the $W$ mass in the limit of high-energy and fixed angle. Consider now, instead, the -+++ amplitude, we would expect the same scaling with the energy but instead we find

$$
\mathcal{A}_{-}=\frac{m^{2}}{E^{2}} \cdot \frac{3 g^{2} \cos ^{2}(\theta / 2)}{2},
$$

which is suppressed by an additional factor $m^{2} / E^{2}$. This is one example of the "helicityinduced" cancellations we mentioned in section 3.3: the on-shell amplitude is anomalously 
reduced with respect to its power counting estimate and there is no reason why this reduction should persist also in the case of off-shell external states. This potentially constitutes a problem for our derivation of the EWA formula because it could lead to an enhancement of the relative corrections, as explained in section 3.3. Actually, the cancellation in equation (3.46) could have been guessed by remembering the usual helicity cancellations in massless gauge theories. By crossing the - - - amplitude and making all the external lines initial we obtain -+++ , since the latter must vanish in the massless limit, there should be a reduction for $m \ll E$. Notice that this reduction factor must be an even power of $m / E$, because of the $m \rightarrow-m$ selection rule of eq. (3.35). Again, because of that selection rule, the $0+++$ amplitude must be odd under $m \rightarrow-m$ and indeed we find

$$
\mathcal{A}_{0}=\frac{m}{E} \cdot \frac{g^{2} \cot (\theta / 2)}{\sqrt{2}} .
$$

The suppression in this case is perfectly understood by power-counting, therefore it must persist also off-shell and does not signal any worrisome on-shell cancellation.

However the helicity induced cancellation which we discovered in eq. (3.46) is not very dangerous. In particular it does not invalidate our derivation of the EWA for the process $q W_{p_{3}}^{-} \rightarrow q^{\prime} W_{p_{3}}^{+} W_{p_{3}}^{-}$, with $\left\{p_{1}, p_{2}, p_{3}\right\}=\{1,1,1\}$, where the external helicities are +++ . In that case, all the three amplitudes $\mathcal{A}_{ \pm}$and $\mathcal{A}_{0}$ appear, corresponding to the possible helicities of the intermediate equivalent $W$. The leading contribution to the total $2 \rightarrow 3$ amplidute comes from just $\mathcal{A}_{+}$in eq. (3.45) while the others, and in particular $\mathcal{A}_{-}$, are subleading precisely because they are canceled. The on-shell cancellation simply implies that we cannot control the off-shell corrections to $\mathcal{A}_{-}$, but these are irrelevant because they are at most as big as $\mathcal{A}_{-}$, and anyhow subleading with respect to $\mathcal{A}_{+}$. In practice, the derivation is saved by the $\mathcal{A}_{+}$term, which is leading and not canceled. Consider now instead the -++ process, $\left\{p_{1}, p_{2}, p_{3}\right\}=\{-1,1,1\}$, for which the three sub-amplitudes read

$$
\begin{aligned}
& \mathcal{A}_{+}=\frac{m^{2}}{E^{2}} \cdot \frac{3 g^{2} \cos ^{2}(\theta / 2)}{2}, \\
& \mathcal{A}_{0}=\frac{m^{3}}{E^{3}} \cdot \frac{g^{2}\left(-4+m_{H}^{2} / m^{2}+9 \cos \theta\right)}{8 \sqrt{2} \tan (\theta / 2)} \\
& \mathcal{A}_{-}=\frac{m^{4}}{E^{4}} \cdot \frac{g^{2}\left(2 m_{H}^{2} / m^{2}+9 \cos \theta-1 / 2(3+\cos \theta) \csc ^{2}(\theta / 2)\right)}{16} .
\end{aligned}
$$

The complicated trigonometric structure of the above equations and also the dependence on the Higgs- $W$ mass ratio $m_{H} / m$, which we take momentarily to be of order one, do not play any role in the following discussion, for which we just need to focus on the $m / E$ dependence of the various terms. Denoting $\varepsilon=m / E$, we see that $\mathcal{A}_{+}$and $\mathcal{A}_{-}$scale respectively as $\varepsilon^{2}$ and $\varepsilon^{4}$ while they were expected from power counting to be of order $\varepsilon^{0}$. The suppression of both $\mathcal{A}_{+}$and $\mathcal{A}_{-}$can again be understood in terms of the amplitude cancellations in massless gauge theories, after crossing, these amplitudes become respectively -+-- and - - - . The occurrence of the double cancellation in $\mathcal{A}_{-}$is instead unexplained. ${ }^{7}$ For what concerns the validity of the EWA, the problem with the -++ process is that both

\footnotetext{
${ }^{7}$ Notice that also the longitudinal amplitude $\mathcal{A}_{0}$ is suppressed in eq. (3.48), it scales like $\varepsilon^{3}$ instead than
} 
the polarized subamplitudes that are expected to lead by power counting are canceled! Because of that cancellation we cannot control the size of the corrections, which could, in principle, be as big as the leading order, thus invalidating the EWA. Of course, the price of the multiple cancellation is that the total amplitude is also canceled so that the potential failure of the EWA in this particular helicity channel, which is subleading, would not show up as a violation of the EWA if we compute only the unpolarized cross section. The dominant helicity amplitudes, such as the +++ previously discussed, are by definition not canceled, and the derivation of the EWA applies without caveats.

The message is then clear: our derivation of EWA, as we presented it in the previous sections, does apply to polarized processes where at least one subamplitude is not suppressed, and, a fortiori, for the total unpolarized cross section. However, at least in its present formulation, our proof does not apply to polarized processes where a cancellation occurs in all intermediate channels. Looking at table 1, where we collected the scaling with $\varepsilon=m / E$ of all the helicity amplitudes, we find that, up to parity and charge conjugation, the polarized processes where our proof fails are,$-++ 0++, 00+$ and $-0+$. The same caveat could apply, a priori, also to the $q \bar{q} \rightarrow q^{\prime} \bar{q}^{\prime} W_{p_{1}}^{+} W_{p_{2}}^{-}$polarized $2 \rightarrow 4$ processes. However, as table 1 shows, it never happens that all the helicity channels associated to the two equivalent $W$ 's cancels simultaneously, there is always at least one channel which is not canceled, i.e. of order $\varepsilon^{0}$.

We stress that the above discussion only shows that our derivation is not valid in some case, and not that the EWA must necessarily fail. It is not excluded that some other mechanism, not taken into account in our approach, like for instance a cancellation affecting the total (exact) $2 \rightarrow 3$ amplitude, suppresses also the corrections by the same amount as the on-shell EWA amplitudes. To investigate the status of the EWA in the potentially problematic channels we performed an explicit computation of $\delta_{E W A}$ along the lines of the computation presented in section 2.2. The result is presented in figure 9 which shows that EWA is satisfied also by those $2 \rightarrow 3$ helicity channels where the leading sub-amplitude is suppressed. We find this rather interesting because it means that the diagrammatic methods employed in the previous section, in which the origin of helicity induced cancellations is not transparent, does not capture entirely the essence of the EWA and the reasons for its validity. This suggests that it might be worth looking for a nondiagrammatic proof of the EWA, which would encompass also the "anomalous" processes.

Finally, we would like to use $W W$ scattering to illustrate the possible enhancement of the subleading corrections via a large ratio of couplings $b$, as discussed in the previous section. Consider the $0+0$ process, the subamplitudes are

$$
\begin{aligned}
& \mathcal{A}_{+}=g^{2} \cot ^{2}(\theta / 2) \\
& \mathcal{A}_{0}=-\frac{m}{E} \cdot \frac{g^{2}\left(2+m_{H}^{2} / m^{2}+3 \cos \theta\right)}{4 \sqrt{2} \tan (\theta / 2)} \\
& \mathcal{A}_{-}=\frac{m^{2}}{E^{2}} \cdot \frac{g^{2}\left(4+m_{H}^{2} / m^{2}+9 \cos \theta\right)}{8}
\end{aligned}
$$

$\varepsilon$. This cancellation cannot be inferred by the standard MHV cancellations in the massless theory because it involves the longitudinal polarizations. It corresponds, in the massless theory, to a cancellation of the Goldstone amplitude with three gauge fields of - - - helicity (after crossing). 


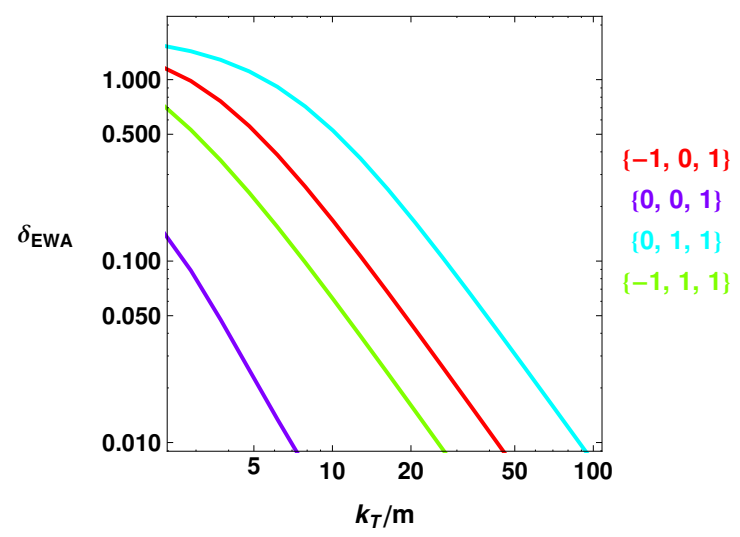

Figure 9. Accuracy of EWA for the $2 \rightarrow 3$ process in eq. (2.12) as a function of $k_{T} / m$ for fixed kinematics given by eq. (2.20). The helicities of the external $W$ bosons are those that result in an anomalous suppression of the sub-amplitudes involved in EWA as shown in table 1. The helicity configuration in each process is $\left\{\lambda\left(W_{\text {in }}^{-}\right), \lambda\left(W_{\text {out }}^{+}\right), \lambda\left(W_{\text {out }}^{-}\right)\right\}$as indicated by the colored labels at the right of the plot.

\begin{tabular}{|c|c|c|c|c|c|c|c|c|c|c|c|}
\hline \multicolumn{3}{|c|}{$W_{+}^{\text {out }}, W_{-}^{\text {out }}=++$} & \multicolumn{3}{|c|}{$W_{+}^{\text {out }}, W_{-}^{\text {out }}=00$} & \multicolumn{3}{|c|}{$W_{+}^{\text {out }}, W_{-}^{\text {out }}=+,-$} & \multicolumn{3}{|c|}{$W_{+}^{\text {out }}, W_{-}^{\text {out }}=0+$} \\
\hline$W_{+}^{\text {in }}$ & $W_{-}^{\text {in }}$ & Scaling & $W_{+}^{\text {in }}$ & $W_{-}^{\text {in }}$ & Scaling & $W_{+}^{\text {in }}$ & $W_{-}^{\text {in }}$ & Scaling & $W_{+}^{\text {in }}$ & $W_{-}^{\text {in }}$ & Scaling \\
\hline+ & + & $\varepsilon^{0}$ & + & + & $\varepsilon^{2}$ & + & + & $\varepsilon^{2}$ & + & + & $\varepsilon$ \\
\hline 0 & + & $\varepsilon$ & 0 & + & $\varepsilon$ & 0 & + & $\varepsilon$ & 0 & + & $\varepsilon^{0}$ \\
\hline- & + & $\varepsilon^{2}$ & - & + & $\varepsilon^{0}$ & - & + & $\varepsilon^{0}$ & - & + & $\varepsilon$ \\
\hline+ & 0 & $\varepsilon$ & + & 0 & $\varepsilon$ & + & 0 & $\varepsilon$ & + & c & $\varepsilon^{2}$ \\
\hline 0 & 0 & $\varepsilon^{2}$ & 0 & 0 & $\varepsilon^{0}$ & 0 & 0 & $\varepsilon^{0}$ & 0 & c & $\varepsilon$ \\
\hline- & 0 & $\varepsilon^{3}$ & - & 0 & $\varepsilon$ & - & 0 & $\varepsilon$ & - & 0 & $\varepsilon^{2}$ \\
\hline+ & - & $\varepsilon^{2}$ & + & - & $\varepsilon^{0}$ & + & - & $\varepsilon^{0}$ & + & - & $\varepsilon$ \\
\hline 0 & - & $\varepsilon^{3}$ & 0 & - & $\varepsilon$ & 0 & - & $\varepsilon$ & 0 & - & $\varepsilon^{2}$ \\
\hline- & - & $\varepsilon^{4}$ & - & - & $\varepsilon^{2}$ & - & - & $\varepsilon^{2}$ & - & - & $\varepsilon^{3}$ \\
\hline
\end{tabular}

Table 1. The table shows the scaling of the polarized amplitudes with the parameter $\varepsilon=m / E$ for $\varepsilon \rightarrow 0$. In the limit, the Higgs mass $m_{H}$ is kept constant and of order $m$. The missing combinations can be obtained by exploiting the $C$ and $P$ symmetry of the $W$ lagrangian.

Apart from the by now habitual $m^{2} / E^{2}$ cancellation which affects $\mathcal{A}_{-}$, the peculiarity of this channel is that the Higgs quadrilinear coupling $\lambda$, which appears through the ratio $m_{H}^{2} / m^{2} \simeq \lambda / g^{2} \equiv b$, does not contribute to all the subprocesses. In particular, it does not contribute to the leading process $\mathcal{A}_{+}$. Therefore, if $b \gg 1$, the subleading process $\mathcal{A}_{0}$ is enhanced and this results in an enhancement of the relative corrections as explained in 
section 3.4. Equation (3.37) reads, in this particular case,

$$
\frac{\mathcal{A}_{0}}{\mathcal{A}_{+}} \simeq \frac{m}{E} b=\frac{m_{H}^{2}}{E m},
$$

which could be made parametrically large in the hypothetical situation of a very light $W$ boson.

\section{Conclusions and outlook}

In this paper we have discussed how, in an ideal experimental situation, it is possible to access the scattering process of on-shell equivalent $W$ bosons disentangling it from the complete partonic interaction. We have shown that this can be achieved in the kinematic regime of forward energetic jets, where the complete process factorizes as a hard scattering convoluted with the collinear emision of the equivalent $W$ boson. This is of course nothing but a statement on the validity of EWA, which we have found to hold up to corrections that scale quadratically with the hardness $H$ of the $W$ interaction. Quantitatively, the relative deviations can be typically estimated as $\operatorname{Max}\left[p_{\perp}^{2} / H^{2}, m^{2} / H^{2}\right]$, with $p_{\perp}$ the forward jet transverse momentum. Actually, we have also found that the corrections could be enhanced, but only in very peculiar situations with, probably, a very limited practical impact.

Our work could be extended in several directions, some of which we will explore in a forthcoming publication. First of all, we should quantify better the level of accuracy of EWA in the practical experimental conditions of the LHC collisions. Notice that the deviations from EWA are regarded, from our viewpoint, as systematic errors in the determination of the equivalent $W$ boson cross section, thus it is crucial that they be kept under control. On the theoretical side, we plan to complement the analysis of the present paper, based on the axial gauge, with a covariant gauge derivation of EWA which presents several interesting aspects. It would also be interesting, in the future, to extend the derivation to higher orders in the perturbative expansion, including in the first place QCD radiative corrections. Another result of our paper deserving further study is the derivation of a generalized EWA formula, eq. (3.31), which provides a prediction of the totally differential cross section. In particular it also describes the distribution of the forward jet azimuthal angle, which is instead integrated over in the standard EWA.

Finally, one interesting aspect of our derivation is that it is definitely not complete because it does not account for the validity of EWA in some peculiar polarized processes, like those listed in section 3.5, which are affected by on-shell suppressions closely analogous to the cancellation of tree-level polarized amplitudes in massless gauge theories. Since the latter ones do not have a clear interpretation in terms of Feynman diagrams, it is not surprising that dealing with such processes becomes cumbersome with our diagrammatic methods. To go beyond, probably, a non-diagrammatic approach would be needed.

\section{Acknowledgments}

We thank C. Anastasiou, A. Ballestrero, A. Banfi, R. Contino, S. Frixione, P. Lodone, Z. Kunszt and D. Zeppenfeld for useful discussions. RF thanks Thomas Hahn for his help 
on the use of the CuBA library. This research is partially supported by the Swiss National Science Foundation under grants 200020-126941 and 200020-138131. The work of RF is also partly supported by the NSF under grants PHY-0910467 and PHY-0652363, and by the Maryland Center for Fundamental Physics. This research was supported in part by the European Programme Unification in the LHC Era, contract PITN-GA-2009-237920 (UNILHC) and by the ERC Advanced Grant no.267985 Electroweak Symmetry Breaking, Flavour and Dark Matter: One Solution for Three Mysteries (DaMeSyFla).

\section{References}

[1] M.S. Chanowitz and M.K. Gaillard, The TeV physics of strongly interacting W's and Z's, Nucl. Phys. B 261 (1985) 379 [inSPIRE].

[2] M.S. Chanowitz and M.K. Gaillard, Multiple production of $W$ and $Z$ as a signal of new strong interactions, Phys. Lett. B 142 (1984) 85 [INSPIRE].

[3] J. Bagger et al., CERN LHC analysis of the strongly interacting $W W$ system: gold plated modes, Phys. Rev. D 52 (1995) 3878 [hep-ph/9504426] [InSPIRE].

[4] J. Bagger et al., The strongly interacting $W W$ system: gold plated modes, Phys. Rev. D 49 (1994) 1246 [hep-ph/9306256] [INSPIRE].

[5] C. Englert, B. Jager, M. Worek and D. Zeppenfeld, Observing strongly interacting vector boson systems at the CERN Large Hadron Collider, Phys. Rev. D 80 (2009) 035027 [arXiv:0810.4861] [INSPIRE].

[6] V.D. Barger, K.-m. Cheung, T. Han and D. Zeppenfeld, Single forward jet tagging and central jet vetoing to identify the leptonic $W W$ decay mode of a heavy Higgs boson, Phys. Rev. D 44 (1991) 2701 [Erratum ibid. D 48 (1993) 5444] [INSPIRE].

[7] A. Ballestrero, D.B. Franzosi and E. Maina, Vector-vector scattering at the LHC with two charged leptons and two neutrinos in the final state, JHEP 06 (2011) 013 [arXiv:1011.1514] [INSPIRE].

[8] A. Ballestrero, G. Bevilacqua and E. Maina, A complete parton level analysis of boson-boson scattering and electroweak symmetry breaking in $l v+$ four jets production at the LHC, JHEP 05 (2009) 015 [arXiv: 0812.5084] [INSPIRE].

[9] J. Butterworth, B. Cox and J.R. Forshaw, WW scattering at the CERN LHC, Phys. Rev. D 65 (2002) 096014 [hep-ph/0201098] [INSPIRE].

[10] K. Doroba et al., The $W_{L} W_{L}$ scattering at the LHC: improving the selection criteria, arXiv: 1201.2768 [INSPIRE].

[11] ATLAS collaboration, G. Aad et al., Expected Performance of the ATLAS experiment Detector, trigger and physics, arXiv:0901.0512 [INSPIRE].

[12] R. Contino, C. Grojean, M. Moretti, F. Piccinini and R. Rattazzi, Strong double Higgs production at the LHC, JHEP 05 (2010) 089 [arXiv:1002.1011] [INSPIRE].

[13] E. Fermi, On the theory of collisions between atoms and electrically charged particles, Nuovo Cim. (19??) 2,143 [hep-th/0205086] [INSPIRE].

[14] E. Williams, Nature of the high-energy particles of penetrating radiation and status of ionization and radiation formulae, Phys. Rev. 45 (1934) 729 [INSPIRE]. 
[15] C. von Weizsacker, Radiation emitted in collisions of very fast electrons, Z. Phys. 88 (1934) 612 [INSPIRE].

[16] S. Dawson, The effective W approximation, Nucl. Phys. B 249 (1985) 42 [INSPIRE].

[17] G.L. Kane, W. Repko and W. Rolnick, The effective $W^{ \pm}, Z_{0}$ approximation for high-energy collisions, Phys. Lett. B 148 (1984) 367 [INSPIRE].

[18] R. Cahn and S. Dawson, Production of very massive Higgs bosons, Phys. Lett. B 136 (1984) 196 [Erratum ibid. B 138 (1984) 464] [INSPIRE].

[19] R.N. Cahn, Production of heavy higgs bosons: comparisons of exact and approximate results, Nucl. Phys. B 255 (1985) 341 [Erratum ibid. B 262 (1985) 744] [INSPIRE].

[20] G. Altarelli, B. Mele and F. Pitolli, Heavy Higgs production at future colliders, Nucl. Phys. B 287 (1987) 205 [INSPIRE].

[21] J. Gunion, J. Kalinowski and A. Tofighi-Niaki, Exact $f f \rightarrow f f W W$ calculation for the charged current sector and comparison with the effective $W$ approximation, Phys. Rev. Lett. 57 (1986) 2351 [INSPIRE].

[22] J. Lindfors, Distribution functions for heavy vector bosons inside colliding particle beams, $Z$. Phys. C 28 (1985) 427 [INSPIRE].

[23] P. Johnson, F.I. Olness and W.-K. Tung, The effective vector boson method for high-energy collisions, Phys. Rev. D 36 (1987) 291 [inSPIRE].

[24] I. Kuss and H. Spiesberger, Luminosities for vector boson-vector boson scattering at high-energy colliders, Phys. Rev. D 53 (1996) 6078 [hep-ph/9507204] [InSPIRE].

[25] R. Kleiss and W.J. Stirling, Anomalous high-energy behavior in boson fusion, Phys. Lett. B 182 (1986) 75 [INSPIRE].

[26] E. Accomando, A. Ballestrero, A. Belhouari and E. Maina, Isolating vector boson scattering at the LHC: gauge cancellations and the equivalent vector boson approximation vs. complete calculations, Phys. Rev. D 74 (2006) 073010 [hep-ph/0608019] [INSPIRE].

[27] A. Alboteanu, W. Kilian and J. Reuter, Resonances and unitarity in weak boson scattering at the LHC, JHEP 11 (2008) 010 [arXiv:0806.4145] [INSPIRE].

[28] Z. Kunszt and D.E. Soper, On the validity of the effective $W$ approximation, Nucl. Phys. B 296 (1988) 253 [INSPIRE].

[29] F. Caravaglios, M.L. Mangano, M. Moretti and R. Pittau, A new approach to multijet calculations in hadron collisions, Nucl. Phys. B 539 (1999) 215 [hep-ph/9807570] [InSPIRE].

[30] M.L. Mangano, M. Moretti and R. Pittau, Multijet matrix elements and shower evolution in hadronic collisions: $W b \bar{b}+n$ jets as a case study, Nucl. Phys. B 632 (2002) 343 [hep-ph/0108069] [INSPIRE].

[31] M.L. Mangano, M. Moretti, F. Piccinini, R. Pittau and A.D. Polosa, ALPGEN, a generator for hard multiparton processes in hadronic collisions, JHEP 07 (2003) 001 [hep-ph/0206293] [INSPIRE].

[32] T. Stelzer and W. Long, Automatic generation of tree level helicity amplitudes, Comput. Phys. Commun. 81 (1994) 357 [hep-ph/9401258] [INSPIRE].

[33] F. Maltoni and T. Stelzer, MadEvent: automatic event generation with MadGraph, JHEP 02 (2003) 027 [hep-ph/0208156] [INSPIRE]. 
[34] W. Kilian, T. Ohl and J. Reuter, WHIZARD: simulating multi-particle processes at LHC and ILC, Eur. Phys. J. C 71 (2011) 1742 [arXiv:0708.4233] [INSPIRE].

[35] M. Moretti, T. Ohl and J. Reuter, O'Mega: an optimizing matrix element generator, hep-ph/0102195 [INSPIRE].

[36] K. Arnold et al., VBFNLO: a parton level Monte Carlo for processes with electroweak bosons, Comput. Phys. Commun. 180 (2009) 1661 [arXiv:0811.4559] [InSPIRE].

[37] K. Arnold et al., VBFNLO: a parton level Monte Carlo for processes with electroweak bosons - Manual for version 2.5.0, arXiv:1107.4038 [INSPIRE].

[38] T. Hahn, Generating Feynman diagrams and amplitudes with FeynArts 3, Comput. Phys. Commun. 140 (2001) 418 [hep-ph/0012260] [INSPIRE].

[39] T. Hahn and M. Pérez-Victoria, Automatized one loop calculations in four-dimensions and D-dimensions, Comput. Phys. Commun. 118 (1999) 153 [hep-ph/9807565] [InSPIRE].

[40] T. Hahn and M. Rauch, News from FormCalc and Loop Tools, Nucl. Phys. Proc. Suppl. 157 (2006) 236 [hep-ph/0601248] [INSPIRE].

[41] T. Hahn, CUBA: a library for multidimensional numerical integration, Comput. Phys. Commun. 168 (2005) 78 [hep-ph/0404043] [INSPIRE]. 